\title{
Structural sensitivities of soft and steep nonlinear global modes in spatially developing media
}

\author{
Yongyun Hwang* \\ Civil and Environmental Engineering, Imperial College London, South Kensington, SW7 2AZ, London, UK
}

\begin{abstract}
Structural sensitivities of soft and steep nonlinear global modes arising in a complex GinzburgLandau model are investigated by calculating the leading-order variation of their amplitude and frequency to an open-loop forcing and a closed-loop perturbation. The soft global mode is found to be sensitive to both the open-loop and closed perturbations in the region of linear absolute instability where its amplitude is not negligible. In particular, the frequency of the soft global mode exhibits large sensitivity at the location where the frequency of soft global mode was shown to be determined in previous WKBJ analysis. On the other hand, the steep global mode shows a large response in the amplitude and the frequency to the open-loop perturbation located far upstream. To the closed-loop perturbation, the steep global mode is most sensitive at the location where the stationary front is located, consistent with the previous WKBJ analysis. Finally, the sensitivities analyzed for the fully nonlinear global mode are compared with those obtained from a weakly nonlinear analysis. It shows that the weakly nonlinear analysis fails to capture the sensitivity behaviour obtained from fully nonlinear global mode particularly under the strong advection yielding steep global mode.
\end{abstract}

Keywords: Nonlinear global mode, Sensitivity, Adjoint equation

\section{Introduction}

Since introduction of the spatio-temporal analysis of instabilities in idealised parallel flows (i.e. absolute and convective instabilities) (Briggs, 1964; Bers, 1983; Huerre and Monkewitz, 1985, 1990), the globally synchronized nonlinear structures appearing in open shear flows such as wake (Mathis et al., 1984; Provansal et al., 1987), mixing layer (Strykowski and Niccum, 1991), and hot jet (Lesshafft et al., 2006), have been understood in terms of the 'global' mode, which often refers to an eigenstructure resulting from a temporally growing instability over the entire spatial domain. Such a nonlinear structure is often tuned with a well-defined characteristic frequency and remains as a large-scale coherent motion even in fully-developed turbulent flows. Therefore, significant effort has been made for understanding the nature of such structures. The reader may refer to reviews by Huerre (2001) and Chomaz (2005) for further details on this issue.

Theoretical description of the linearly growing global mode has been well established. One may classify it into two categories: local and global approaches. The local approach is based

*e-mail:y.hwang@imperial.ac.uk 
on a WKBJ theory with the assumption that the base flow is weakly non-parallel (i.e. $X=\epsilon x$ where $x$ denotes the streamwise direction with $\epsilon \ll 1$ ). In this circumstance, the evolution of a disturbance obeys the local dispersion relation obtained by setting the base flow at a given streamwise location $X$ to be parallel. The frequency $\omega_{L G}$ of the linearly growing disturbance over the entire flow domain is then determined in terms of local absolute frequency $\omega_{0}(X)$ (Chomaz et al., 1988, 1991; Monkewitz et al., 1993):

$$
\omega_{L G}=\omega_{0}\left(X_{l}^{s}\right) \text { with }\left.\frac{\partial \omega_{0}}{\partial X}\right|_{X=X_{l}^{s}}=0
$$

where $X$ now turns out to be the complex streamwise location. It is important to note that the criterion implies that a finite region of local absolute instability $\left(\omega_{0, i}(X)>0\right)$ is necessary for $\omega_{L G, i}>0$ (Chomaz et al., 1991; Monkewitz et al., 1993), suggesting physical importance of the local absolute instability in generating linear global instability.

In many practical situations, the flow configuration, however, often involves strong nonparallelism and complexity. The linear global mode and its eigenfrequency $\omega_{L G}$ in this case are obtained by numerically solving the global eigenvalue problem. The earliest approach of this type was probably performed by Zebib (1987) and Jackson (1987) who successfully predicted the critical Reynolds number for the onset of Kármán vortex shedding in bluff-body wakes. The use of the adjoint global mode was introduced by Hill (1992) for control of linear global mode, whose work also led Hwang and Choi (2006) with a similar approach for local absolute instability. Giannetti and Luchini (2007) recently revisited the work by Hill (1992) and performed a sensitivity analysis to identify the region where a small localised feedback forcing leads to a large drift of the linear global frequency. They showed that the linear global frequency is highly sensitive if the forcing is located in the region where the regular and adjoint global modes overlap. Importantly, this location is found to be very similar to the region where the presence of a small secondary cylinder stabilizes vortex shedding (Strykowski and Sreenivasan, 1990) as also demonstrated by Marquet et al. (2008). Recently, the computed regular and adjoint global modes have also been used by combining with the classical weakly nonlinear theory, and it has provided much deeper physical insight into the dynamics of global instability particularly when multiple linear global modes compete with each other for nonlinear pattern selection (see also e.g. Meliga et al., 2009, among others).

Despite the encouraging progress, both the local and global approaches with linear global mode are, in principle, valid only in the regime where the role of nonlinearity is weak: i.e. the regime where the bifurcation control parameter such as the Reynolds number is not very far from the onset of the instability. Theoretical effort has therefore been made to describe the global mode in the 'fully' nonlinear regime where the bifurcation control parameter is sufficiently far from the onset. While the local approach with the assumption of parallel or weakly non-parallel flow was maintained, the dynamics of a nonlinear global mode was described in terms of the front propagating stable to unstable state. (Chomaz, 1992; Couairon and Chomaz, 1996, 1997a,b, 1999a,b; Pier and Huerre, 1996; Pier et al., 1998, 2001; Pier and Huerre, 2001a; van Saarloos, 2003). In the fully nonlinear regime, a growing disturbance nucleates into a front, the downstream of which is composed of nonlinear instability waves. As the propagating velocity of the front is often identical to that of the leading and trailing edges of a linear wavepacket (Delbende and Chomaz, 1998; Chomaz, 2003), the local absolute instability in the medium pushes the nucleated front to propagate. The front subsequently loses its velocity at the location where the nature of the local instability transits from convective to absolute $\left(X=X^{c a}\right)$, and settles at this location while generating instability wave downstream. The stationary front therefore acts as a wavemaker, and 
the frequency of the nonlinear global mode $\omega_{N G}$ is given by local 'linear' absolute frequency at $X=X^{c a}$ : i.e.,

$$
\omega_{N G}=\omega_{0}\left(X^{c a}\right) .
$$

The nonlinear global mode, the frequency of which is given by (2), has been referred to as 'steep' or 'elephant' mode due to the sharp stationary front in its spatial structure (see also figure 2 and Pier and Huerre, 2001b). Application of the frequency selection criterion (2) to a number of canonical flows such as wakes (Pier and Huerre, 2001a; Pier, 2002), hot jets (Lesshafft et al., 2006), and swirling vortex (Gallaire et al., 2006) has been shown to be successful.

By definition, the nonlinear global mode is just a nonlinear solution of the time-dependent governing equation, which is easily computed with well-established modern CFD solvers. Therefore, as in the case of linear global mode, one may think of a global approach which identifies the region where a certain type of forcing would yield a large drift of the amplitude and/or the frequency of a nonlinear global mode. The scope of the present study is to address this issue by designing a sensitivity analysis of a nonlinear global mode in the fully nonlinear regime. However, the sensitivity analysis for a nonlinear global mode has been very rarely carried out, and, to the best of my knowledge, only two works have addressed so far: Hwang and Choi (2008) and Luchini et al. (2008). The former computed sensitivity of the amplitude (energy) of the stationary nonlinear global mode in a real Ginzburg-Landau equation, whereas the latter calculated sensitivity of the frequency of the temporally periodic nonlinear global mode (Kármán vortex shedding) in a circular cylinder wake. Unfortunately, the two approaches have not been applied to the same flow configuration, thus it is difficult to see what kind of differences would be yielded depending on the objective functional of interest (i.e. amplitude or frequency). Furthermore, their results have not been well discussed in comparison with the physical insight gained from the previous local analyses using the framework of the front propagation. To fill these gaps, the present study is aimed at applying the two approaches to a supercritical complex Ginzburg-Landau (CGL) equation, which yields nonlinear global modes extensively analyzed by a nonlinear WKBJ analysis (Pier and Huerre, 1996; Pier et al., 1998, 2001).

This paper is organized as follows. In section 2, we briefly introduce linear and nonlinear global modes in the CGL equation with their frequency criterion. In particular, two types of nonlinear global mode respectively called 'soft' and 'steep' modes are illustrated (Pier and Huerre, 1996; Pier et al., 1998, 2001). In section 3, structural sensitivities of the amplitude and the frequency of a nonlinear global mode to small open-loop and closed-loop perturbations are formulated following Hwang and Choi (2008) and Luchini et al. (2008). The computed sensitivities are then shown in section 4 with a discussion. In particular, we compare them with those obtained using a weakly nonlinear analysis. Finally, a summary and a few remarks are given in section 5 .

\section{Nonlinear global modes in the complex Ginzburg-Landau equation} 2001):

We consider a complex Ginzburg-Landau equation given in the following form (Pier et al.,

$$
i \frac{\partial \psi}{\partial t}=\left(\omega_{0}(x)+\frac{1}{2} \omega_{k k} k_{0}^{2}\right) \psi+i \omega_{k k} k_{0} \frac{\partial \psi}{\partial x}-\frac{1}{2} \omega_{k k} \frac{\partial^{2} \psi}{\partial x^{2}}+\gamma|\psi|^{2} \psi,
$$

with boundary condition

$$
\psi(x= \pm \infty)=0 .
$$


Here, $\psi(x, t)$ is the complex state function with the streamwise direction $x \in(-\infty, \infty)$ and the time $t \in[0, \infty)$. The parameters $k_{0}, \omega_{0}(x), \omega_{k k}$ and $\gamma$ are set to be complex. We note that this form of the Ginzburg-Landau equation is convenient for the local stability analysis, as we shall see in section 2.1 where the details of the parameters, $k_{0}, \omega_{0}$, and $\omega_{k k}$ are given. For simplicity, we set $k_{0}, \omega_{k k}$, and $\gamma$ to be constant, while $\omega_{0}(x)$ is considered to vary along $x$ such that:

$$
\omega_{0}(x)=\omega_{0}^{\max }+\frac{1}{2} \omega_{0 x x} x^{2},
$$

where $\omega_{0 x x}$ is a complex constant. It should be mentioned that $\omega_{0}(x)$ here is purposely set as in (4) to avoid the term depending linearly on $x$ (see also section 2.2.2 for further discussion).

In setting the coefficients of (3), there are some requirements to ensure well-posedness of (3). It is useful to rearrange (3) as

$$
\frac{\partial \psi}{\partial t}+U \frac{\partial \psi}{\partial x}=\mu(x) \psi+D \frac{\partial^{2} \psi}{\partial x^{2}}-i \gamma|\psi|^{2} \psi
$$

where

$$
U \equiv-\omega_{k k} k_{0}, \quad \mu(x) \equiv-i\left(\omega_{0}(x)+\frac{1}{2} \omega_{k k} k_{0}^{2}\right), \quad D \equiv \frac{i}{2} \omega_{k k} .
$$

This form suggests that $U$ can be interpreted as complex advection velocity, $\mu(x)$ a function controlling local instability property, and $D$ the complex diffusivity where its imaginary part acts for dispersion. First, to restrict our discussion only to the case of downstream advection, it is necessary to set $U_{r} \geq 0$, giving $k_{0, i} \leq 0$. Also, the well-posedness of (3) with its boundary condition $(\psi(x= \pm \infty)=0)$ would require any perturbations in $\psi$ to decay at $x= \pm \infty$, yielding $\omega_{0 x x, i}<0$. The positive diffusivity $\left(D_{r}>0\right)$, which also ensures the causality of (3), gives $\omega_{k k, i}<0$. Finally, to obtain stably sustaining nonlinear global modes, the nonlinear term should be stabilizing, requiring $\gamma_{i}<0$.

\subsection{Linear global instability}

Linear global stability analysis of (3) has been performed by a number of early studies (e.g. Chomaz et al., 1988; Huerre and Monkewitz, 1990; Chomaz et al., 1991; Le Dizés et al., 1996; Huerre and Rossi, 1998). Here, we therefore briefly discuss the main results. Equation (3) admits a solution $\psi_{0}(x, t)=0$ for all the parameters, setting it as a basic state of (3). We now consider a small perturbation around this basic state: $\psi(x, t)=\psi_{0}+\epsilon \psi^{\prime}(x, t)+O\left(\epsilon^{2}\right)$. Then, at $O(\epsilon)$, (3) becomes

$$
\frac{\partial \psi^{\prime}}{\partial t}=L \psi^{\prime}
$$

where

$$
L \equiv \omega_{k k} k_{0} \frac{\partial}{\partial x}-i\left(\omega_{0}(x)+\frac{1}{2} \omega_{k k} k_{0}^{2}\right)+\frac{i}{2} \omega_{k k} \frac{\partial^{2}}{\partial x^{2}} .
$$

We seek a solution of (6) in the form of $\psi^{\prime}(x, t)=\delta \phi_{L G}(x) e^{-i \omega_{L G} t}$, yielding the following eigenvalue problem:

$$
-i \omega_{L G} \delta \phi_{L G}=L \delta \phi_{L G},
$$

where $\delta \phi_{L G}(x)$ and $\omega_{L G}$ are respectively the linear global mode and its complex frequency. Exact analytic solution of (7) is available as we shall see in section 4.5. However, for illustrative purpose, here we perform a local stability analysis based on the standard WKBJ theory (see also 
Huerre and Monkewitz, 1990; Le Dizés et al., 1996; Huerre and Rossi, 1998, for further details). Under the assumption that $\omega_{0}(x)$ varies slowly along the streamwise direction (i.e. $\omega_{0 x x}=\epsilon^{2} \omega_{0 X X}$ with $\epsilon \ll 1$ and $\left.\omega_{0 X X} \sim O(1)\right)$, the leading-order local dispersion relation of (7) is obtained by setting $\psi^{\prime}(x, t)=A(X) \exp \left(i / \epsilon \int^{X} k_{l}(X ; \omega) d X-i \omega t\right)+c . c(A(X)$ is the amplitude of the linear global mode) as

$$
\omega=\Omega_{l}(k(X), X),
$$

where

$$
\Omega_{l}(k(X), X)=\omega_{0}(X)+\frac{1}{2} \omega_{k k}\left(k(X)-k_{0}\right)^{2} .
$$

We note that $\omega=\omega_{0}(X)$ is obtained if $k=k_{0}$ at which $\partial \omega /\left.\partial k\right|_{k=k_{0}}=0$, indicating that $\omega_{0}(X)$ and $k_{0}$ are respectively the local absolute frequency and absolute wavenumber. Also, from (8b), $\omega_{k k}=\left.\partial_{k k} \omega\right|_{k=k_{0}}$.

Applying the frequency selection criterion (1) to (8) gives the linear global frequency at $O(\epsilon)$ (Huerre and Monkewitz, 1990; Huerre and Rossi, 1998):

$$
\omega_{L G}=\omega_{0}^{\max } .
$$

This indicates that if $\omega_{0, i}^{\max }>0$, the basic state becomes globally linearly unstable. For convenience, from now on, we shall consider $\omega_{0, i}^{\max }$ as a bifurcation control parameter such as the Reynolds number (see also sections 4.1 and 4.2).

\subsection{Nonlinear global modes}

If the basic state becomes globally linearly unstable, a disturbance grows temporally and experiences the stabilizing nonlinearity. It then settles down to a limit-cycle oscillation, forming a nonlinear global mode. The weakly nonlinear theory would probably be the first step to be taken to analyze such a global mode. However, it has been shown that a theoretical difficulty arises because the parameter space where the weakly nonlinear theory is valid becomes highly limited in weakly non-parallel medium (Le Dizés et al., 1993; Chomaz, 2005). Theoretical description of nonlinear global mode has therefore been made by taking the nonlinearity of the system fully into account. There have been two types of theoretical approaches, one of which is by analyzing the structure of a nonlinear global mode in the phase plane to tackle the nonlinear eigenvalue problem associated with its frequency selection (e.g. Couairon and Chomaz, 1996, 1997a,b, 1999a), and the other is based on a nonlinear WKBJ theory (Pier and Huerre, 1996; Pier et al., 1998, 2001; Pier and Huerre, 2001a). The nonlinear global modes in the CGL equation (3) was intensively analyzed particularly by the latter, hence we follow it to introduce the nonlinear global modes.

If the basic state $\psi_{0}(x, t)=0$ becomes linearly or nonlinear unstable, equation (3) admits a non-trivial solution $\psi(x, t)=\psi_{N G}(x, t)$, referred to as nonlinear global mode, in the form of

$$
\psi_{N G}(x, t)=\phi_{N G}(x) e^{-i \omega_{N G} t},
$$

where $\phi_{N G}(x)$ is a complex-valued function describing the spatial structure of the nonlinear global mode and $\omega_{N G}$ is its 'real' frequency. As mentioned, a nonlinear WKBJ theory was used to describe the nonlinear global mode when $\omega_{0}(X)$ varies slowly along the streamwise direction (Pier and Huerre, 1996; Pier et al., 1998, 2001; Pier and Huerre, 2001a). At the leading order, the linear dispersion relation (8) is used to approximate the nonlinear global mode in the region where the amplitude of the solution is expected to be small. However, in the region of large global-mode amplitude, the solution would experience strong nonlinearity, thus it is approximated in the form 


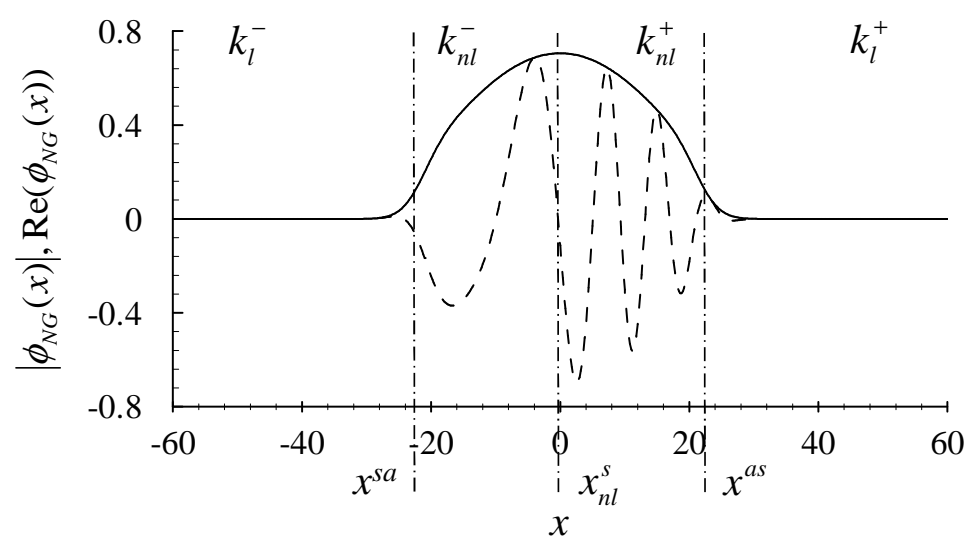

Figure 1: Spatial structure of soft nonlinear global mode $\left(k_{0}=0.5, \omega_{k k}=-i, \omega_{0, \max }=0.4+0.5 i, \omega_{0 x x}=-0.0004-0.002 i\right.$, $\gamma=-i): \stackrel{-}{\longrightarrow},\left|\phi_{N G}(x)\right| ;----, \operatorname{Re}\left(\phi_{N G}(x)\right)$. Here, $x_{n l}^{s}=0, x^{s a}=-22.4$ and $x^{a s}=22.4$, and see the text for their physical implications.

of $\psi \sim \Psi\left(\epsilon^{-1} \int^{X} k_{n l}(X ; \omega) d X-i \omega t+\Theta(X) ; k_{n l}(X, \omega), X\right)$. This yields the following local nonlinear dispersion relation (see also e.g. Pier and Huerre, 1996; Pier et al., 2001):

$$
\omega=\Omega_{n l}\left(k_{n l}(X), X\right)
$$

where

$$
\Omega_{n l}\left(k_{n l}(X), X\right)=\frac{\operatorname{Im}\left(\gamma^{*} \Omega_{l}\left(k_{n l}(X), X\right)\right.}{\operatorname{Im}\left(\gamma^{*}\right)} .
$$

Here, the superscript ${ }^{*}$ is the complex conjugate, and $k_{n l}$ and $\omega$ are respectively the real streamwise wavenumber and the real frequency. At the leading order, the spatial structure of the nonlinear global mode is constructed by (8) and (11), and two types of nonlinear global mode have been found: soft and steep global modes.

\subsubsection{Soft global mode}

Figure 1 shows an example of the soft global mode found by Pier and Huerre (1996) The soft global mode typically appears with relatively weak advection (see also figure 3 ). Within the WKBJ theory, the spatial structure of the mode is roughly divided into four regions, the boundaries of which are respectively given by $x^{s a}, x_{n l}^{s}$ and $x^{a s}$. Here, $x_{n l}^{s}=\epsilon^{-1} X_{n l}^{s}$ (see below for $\left.X_{n l}^{s}\right), x^{s a}$ is the upstream location where the local linear instability changes from linearly stable to absolutely unstable region, and $x^{a s}$ is the downstream location where the local linear instability transits from absolutely unstable to linearly stable region. Here, it should be noted that $\max \left(\operatorname{Im}\left(\Omega_{l}\right)=0\right.$ for every real $k$ at $x=x^{s a}$ and $x=x^{a s}$ because there is no locally convectively unstable region due to the absence of advection.

In both far upstream and downstream $\left(x<x^{s a}\right.$ and $x>x^{a s}$ in figure 1), the amplitude of the global mode is small. Therefore, the spatial structure is dominated by the linear spatial branches (wavenumbers) originating from (8):

$$
k_{l}^{ \pm}(X)=k_{0} \pm \sqrt{\frac{2\left(\omega-\omega_{0}\right)}{\omega_{k k}}},
$$




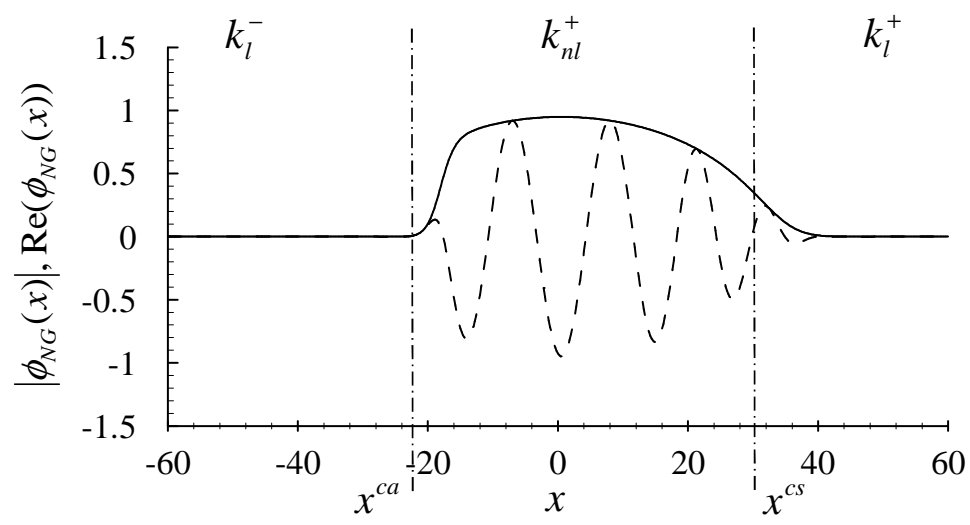

Figure 2: Spatial structure of steep nonlinear global mode $\left(k_{0}=0.5-0.9 i, \omega_{k k}=-i, \omega_{0, \max }=0.4+0.5 i, \omega_{0 x x}=\right.$ $-0.0004-0.002 i, \gamma=-i):-,\left|\phi_{N G}(x)\right| ;----, \operatorname{Re}\left(\phi_{N G}(x)\right)$. Here, $x^{c a}=-22.4$ and $x^{c s}=30.1$, and see the text for their physical implications.

where $k_{l}^{-}$and $k_{l}^{+}$are respectively upstream and downstream spatial branches, which are classified by causality. In the region of $x^{s a}<x<x^{a s}$, the spatial structure is composed of upstream $\left(k_{n l}^{-}\right)$ and downstream $\left(k_{n l}^{+}\right)$nonlinear spatial branches obtained similarly from the nonlinear dispersion relation (11) (see also figure 1). The two nonlinear spatial branches, $k_{n l}^{-}$and $k_{n l}^{+}$, meet each other at $x=x_{n l}^{s}$, at which the leading-order frequency of the soft global mode $\omega_{N G}^{\text {soft }}$ is determined as

$$
\omega_{N G}^{s o f t}=\omega_{n l}^{s} \text { and } \omega_{n l}^{s}=\Omega_{n l}\left(X_{n l}^{s}, k_{n l}^{s}\right),
$$

with

$$
\left.\frac{\partial \omega_{n l}}{\partial X}\right|_{X=X_{n l}^{s}}=\left.\frac{\partial \omega_{n l}}{\partial k}\right|_{k=k^{s}}=0
$$

In the case of (3), an explicit form of $\omega_{N G}^{\text {soft }}$ is given as

$$
\omega_{N G}^{s o f t}=\omega_{0, r}^{\max }-\frac{\gamma_{r}}{\gamma_{i}} \omega_{0, i}^{\max }+\frac{\left|\gamma^{*} \omega_{k k}\right|}{2 \gamma_{i} \operatorname{Im}\left(\gamma^{*} \omega_{k k}\right)} k_{0 i}^{2}
$$

\subsubsection{Steep global mode}

An example of the steep global mode found in Pier et al. (1998) and Pier et al. (2001) is shown in figure 2. The steep global mode typically appears in the presence of strong advection (see also figure 3). Its spatial structure consists of three regions as shown in figure 2, and their boundaries are respectively indicated as $x^{c a}$ and $x^{c s}$. Here, $x^{c a}$ denotes the upstream location where the local linear instability transits from convectively unstable to absolutely unstable region (figure 2), and $x^{c s}$ is the downstream location where the local linear instability changes from convectively unstable to linear stable region: i.e. $\operatorname{Im}\left(\omega_{0}\right)=0$ at $x=x^{c a}$ and $\max \left(\operatorname{Im}\left(\Omega_{l}\right)\right)=0$ for every real $k$ at $x=x^{c s}$.

Similarly to the soft global mode, in both far upstream and downstream $\left(x<x^{c a}\right.$ and $x>$ $x^{c s}$ in figure 2), the amplitude of the global mode is small. Therefore, the spatial structure is dominated by the linear spatial branches $k_{l}^{-}$and $k_{l}^{+}$. However, near $x=x^{c a}$, the structure is characterized with a stationary front contrary to the soft global mode (see the sharp slope right 


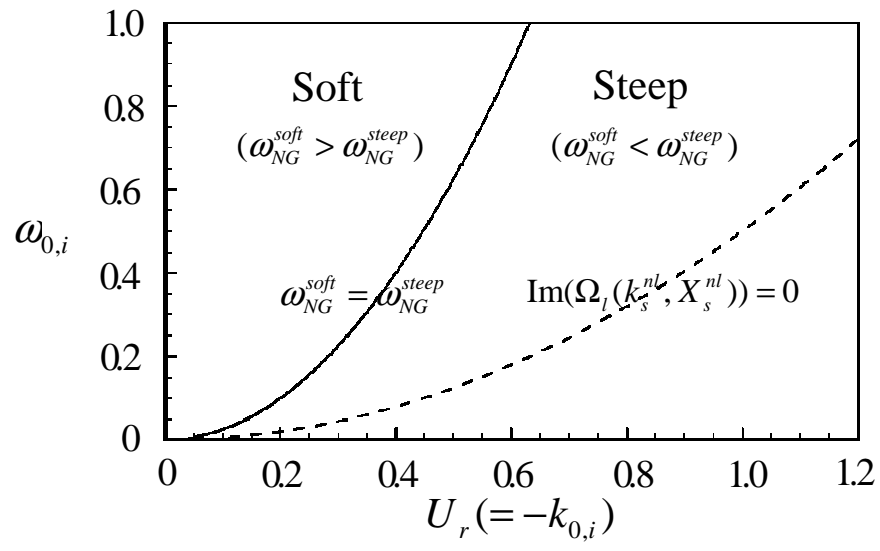

Figure 3: Domains of the existence of steep and soft global modes $\left(k_{0}=0.5, \omega_{k k}=-i, \omega_{0, \max }=0.4+0.5 i, \omega_{0 x x}=\right.$ $-0.0004-0.002 i, \gamma=-i):-, \omega_{N G}^{\text {soft }}=\omega_{N G}^{\text {steep }} ;----, \operatorname{Im}\left(\Omega_{l}\left(k_{s}^{n l}, X_{s}^{n l}\right)=0\right.$. For the given parameters here, the boundary between the steep and the soft global modes is given by $\omega_{N G}^{\text {soft }}=\omega_{N G}^{\text {steep }}$.

next to $x=x^{c a}$ in figure 2). This front directly connects the upstream linear spatial branch $k_{l}^{-}$ to the downstream nonlinear one $k_{n l}^{+}\left(k_{n l}^{-}\right.$branch does not appear in the steep global mode). Pier et al. (2001) showed that the front at $x \simeq x^{c a}$ acts as a wavemaker of the nonlinear global mode and the frequency of the steep global mode $\omega_{N G}^{\text {steep }}$ is determined as

$$
\omega_{N G}^{\text {steep }}=\omega_{0}\left(X^{c a}\right)
$$

where

$$
\omega_{0}\left(X^{c a}\right)=\omega_{0, r}^{\max }-\frac{\omega_{0 x x, r}}{\omega_{0 x x, i}} \omega_{0, i}^{\max } .
$$

Here, it should be mentioned that equation (14a) indicates that the steep global mode appears as soon as $\omega_{0, i}(X)>0$ for any streamwise location $X$ (Pier et al., 2001). On the other hand, the linear global instability generally appears if the spatial domain contains a finite region of local absolute instability (Huerre and Monkewitz, 1990; Chomaz et al., 1991; Huerre and Rossi, 1998). This implies that the steep global mode would be able to emerge even if the basic state is linearly globally stable. This feature appears to be particularly important if $\omega_{0, r}(X)$ contains the term linearly proportional to $X$ (see Pier and Huerre, 2001a, for further details). In the present study, we aim to consider only supercritical bifurcation as in a bluff-body wake for the simplicity. Therefore, we purposely set $\omega_{0}(x)$ as in (4) to inhibit the emergence of the subcritical bifurcation in the CGL equation (3).

\subsubsection{Transition from soft to steep global mode with the advection}

Within the framework of the WKBJ theory, the emergence of soft and steep nonlinear global modes is explicitly determined in the parameter space of the CGL equation (3) (Pier et al., 2001). The criteria are summarized as follows:

1. For $\omega_{0, i}^{\max }<0$, no nonlinear global modes appear and the flow is linearly globally stable.

2. If $\omega_{0, i}^{\max }>0$ and $\Omega_{l, i}\left(k_{n l}^{s}, X_{n l}^{s}\right)<0$, only the steep mode exists and appears. 
3. If $\Omega_{l, i}\left(k_{n l}^{s}, X_{n l}^{s}\right)>0$ and $\omega_{N G}^{\text {soft }}<\omega_{N G}^{\text {steep }}$, both the soft and the steep modes exist but the steep mode is selected.

4. For $\Omega_{l, i}\left(k_{n l}^{s}, X_{n l}^{s}\right)>0$ and $\omega_{N G}^{\text {soft }}>\omega_{N G}^{\text {steep }}$, the soft mode is selected.

Here,

$$
\Omega_{l}\left(k_{n l}^{s}, X_{n l}^{s}\right)=\omega_{0, i}^{\max }+\frac{\left|\omega_{k k}\right|^{2} \operatorname{Im}\left(\gamma^{2} \omega_{k} k^{*}\right)}{2\left(\operatorname{Im}\left(\gamma^{*} \omega_{\mathrm{kk}}\right)\right)^{2}} k_{0, i}^{2} .
$$

According to these criteria, the domain of the existence of soft and steep global modes in the $\omega_{0, i}^{\max }$ and $U_{r}$ plane is described as in figure 3. It should be mentioned that the domain of the existence of the nonlinear global mode is identical to that of the linear global instability at least within the framework of the WKBJ analysis (i.e. $\omega_{L G, i}\left(=\omega_{0, i}^{\max }\right)>0$ ). Figure 3 also indicates that the advection velocity $U_{r}\left(=\operatorname{Re}\left(-\omega_{k k} k_{0}\right)\right.$ plays a critical role in determining the global mode. The soft global mode typically appears when $U_{r}$ is relatively small, whereas the steep global mode prevails for large $U_{r}$.

\section{Structural sensitivities of nonlinear global mode}

The structural sensitivity of a global mode in the linear and/or the weakly non-linear regimes (Chomaz, 2005; Giannetti and Luchini, 2007; Hwang and Choi, 2008) is characterized by its response to small-amplitude perturbation. In the similar vein, we characterize the response of a nonlinear global mode to small perturbations. We start by recasting (3) to which a smallamplitude perturbation $w(x, t)$ is added with the amplitude $a(\ll 1)$ :

$$
\frac{\partial \psi}{\partial t}=L \psi-i \gamma|\psi|^{2} \psi+a w(x, t)
$$

The small perturbation is then set to be composed of in the form of an open-loop and a closedloop forcing without loss of generality:

$$
w(x, t)=f(x, t)+c(x) \psi(x, t) .
$$

Here, the open-loop perturbation may be viewed as a general form of external noise to examine the response of the system whereas the small closed-loop perturbation may be considered as a small error or uncertainty in the intrinsic parameters. The response of the given nonlinear global mode to such perturbation is then quantified by monitoring its amplitude and frequency which will be defined below (see sections 3.1 and 3.2). Here, it should be pointed out that it would probably be most relevant to compare the results of the present sensitivity analysis to the frequency selection criteria given in (13) and (14) from the sensitivity of the frequency to closed-loop perturbation which examines the response of the global-mode frequency to noise in the intrinsic parameters such as $\omega_{0}(x)$.

\subsection{Amplitude}

We first consider the amplitude of a nonlinear global mode. Following Hwang and Choi (2008), we find the amplitude as energy of the global mode such that

$$
A \equiv \lim _{T \rightarrow \infty} \frac{1}{T} \int_{0}^{T} \int_{\Omega}\left|\psi_{N G}(x, t)\right|^{2} d x d t
$$


where $\Omega$ is the streamwise domain of (3). In the absence of the forcing term (i.e. $a=0$ ), the amplitude of the global mode becomes $A=\int_{\Omega}\left|\phi_{N G}(x)\right|^{2} d x$ from (10). The presence of a small perturbation $(a \ll 1)$ would lead the solution of (16) to be written as $\psi(x, t)=\psi_{N G}(x, t)+$ $a \delta \psi(x, t)+O\left(a^{2}\right)$, resulting in $A \rightarrow A+a \delta A+O\left(a^{2}\right)$ where

$$
\delta A \equiv \lim _{T \rightarrow \infty} \frac{1}{T} \int_{0}^{T} \int_{\Omega} \delta \psi(x, t) \psi_{N G}^{*}(x, t)+\delta \psi^{*}(x, t) \psi_{N G}(x, t) d x d t .
$$

At $O(a)$, the perturbed variable $\delta \psi(x, t)$ satisfies the so-called tangent equation of (16) which is essentially the linearized equation about $\psi(x, t)=\psi_{N G}(x, t)$ :

$$
\frac{\partial \delta \psi}{\partial t}=L \delta \psi-i 2 \gamma\left|\psi_{N G}\right|^{2} \delta \psi-i \gamma \psi_{N G}^{2} \delta \psi^{*}+w(x, t),
$$

with initial and boundary conditions,

$$
\delta \psi(x= \pm \infty, t)=0 \text { and } \delta \psi(x, t=0)=0 .
$$

The next step would then be to build a proper adjoint equation of (19) so that the functional $\delta A$ is explicitly given in terms of $w(x, t)$. However, the complex conjugate variable $\delta \psi^{*}$ in (19) yields a difficulty in introducing the adjoint equation. To overcome this difficulty, we first take the complex conjugate to (19):

$$
\frac{\partial \delta \psi^{*}}{\partial t}=L^{*} \delta \psi^{*}+i 2 \gamma^{*}\left|\psi_{N G}\right|^{2} \delta \psi^{*}+i \gamma^{*}\left(\psi_{N G}^{2}\right)^{*} \delta \psi+w^{*}(x, t) .
$$

We then define a vector form of the perturbed state variable such that $\delta \mathbf{q}=\left[\delta \psi \delta \psi^{*}\right]^{T}$ (the superscript ${ }^{T}$ indicates the transpose), enabling us to build the following linearized system:

$$
\mathbf{L} \delta \mathbf{q}=\mathbf{w},
$$

where

$$
\mathbf{L}=\frac{\partial}{\partial t} \mathbf{I}-\left(\begin{array}{cc}
L-i 2 \gamma\left|\psi_{N G}\right|^{2} & -i \gamma \psi_{N G}^{2} \\
i \gamma^{*}\left(\psi_{N G}^{2}\right)^{*} & L^{*}+i 2 \gamma^{*}\left|\psi_{N G}\right|^{2}
\end{array}\right), \quad \mathbf{w}=\left(\begin{array}{c}
w \\
w^{*}
\end{array}\right) .
$$

Here, $\mathbf{I}$ is the identity matrix. We now note that equation (19) turns out to be the standard matrix form of a linear system as in (21). This then allows us to define the adjoint operator using the following standard inner product for arbitrary complex vector variables $\mathbf{f}$ and $\mathbf{g}$ :

$$
\langle\mathbf{f}, \mathbf{g}\rangle=\lim _{T \rightarrow \infty} \frac{1}{T} \int_{0}^{T} \int_{\Omega} \mathbf{f}^{H} \mathbf{g} d x d t,
$$

where the superscript ${ }^{H}$ is the complex conjugate transpose. We note that this leads us to write the leading-order variation of the amplitude as

$$
\delta A=\left\langle\mathbf{q}_{N G}, \delta \mathbf{q}\right\rangle,
$$

where $\mathbf{q}_{N G}=\left[\psi_{N G} \psi_{N G}^{*}\right]^{T}$.

The relation to define the adjoint operator with respect to the inner product (22) is then given as

$$
\left\langle\delta \mathbf{q}_{a}^{+}, \mathbf{L} \delta \mathbf{q}\right\rangle=\left\langle\mathbf{L}^{+} \delta \mathbf{q}_{a}^{+}, \delta \mathbf{q}\right\rangle,
$$


where

$$
\mathbf{L}^{+}=-\frac{\partial}{\partial t} \mathbf{I}-\left(\begin{array}{cc}
L^{+}+i 2 \gamma^{*}\left|\psi_{N G}\right|^{2} & -i \gamma \psi_{N G}^{2} \\
i \gamma^{*}\left(\psi_{N G}^{2}\right)^{*} & L^{+*}-i 2 \gamma\left|\psi_{N G}\right|^{2}
\end{array}\right)
$$

with

$$
L^{+}=-\omega_{k k}^{*} k_{0}^{*} \frac{\partial}{\partial x}+i\left(\omega_{0}^{*}(x)+\frac{1}{2} \omega_{k k}^{*} k_{0}^{* 2}\right)-\frac{i}{2} \omega_{k k}^{*} \frac{\partial^{2}}{\partial x^{2}} .
$$

Here, the superscript ${ }^{+}$denotes the adjoint operator and variable, and the vector form of the adjoint variable is defined as $\delta \mathbf{q}_{a}^{+} \equiv\left[\delta \psi_{a}^{+} \delta \psi_{a}^{+*}\right]^{T}$. We also note that, in (24a), the contribution of the boundary conditions is not included as it will disappear by setting the proper terminal and boundary conditions of $\delta \mathbf{q}_{a}^{+}$(see also (25b)).

Using the adjoint operator, we now build the following equation:

$$
\mathbf{L}^{+} \delta \mathbf{q}_{a}^{+}=\mathbf{q}_{N G},
$$

with the terminal and boundary conditions,

$$
\delta \mathbf{q}_{a}^{+}(x, t=\infty)=0 \text { and } \delta \mathbf{q}_{a}^{+}(x= \pm \infty, t)=0 .
$$

Here, we note that the first row of the matrix equation (25), which is essentially the same as that of the second row, gives

$$
-\frac{\partial \delta \psi_{a}^{+}}{\partial t}=L^{+} \delta \psi_{a}^{+}+i 2 \gamma^{*}\left|\psi_{N G}\right|^{2} \delta \psi_{a}^{+}-i \gamma \psi_{N G}^{2} \delta \psi_{a}^{+*}+\psi_{N G}(x, t)
$$

Since $\psi_{N G}(x, t)=\phi_{N G}(x) e^{-i \omega_{N G} t}$ from (10), the Floquet's theory leads the solution of (26) to take the following form for $t \rightarrow 0$ :

$$
\delta \psi_{a}^{+}(x, t)=\delta \phi_{a}^{+}(x) e^{-i \omega_{N G} t},
$$

where $\delta \phi_{a}^{+}(x)$ is the function describing the spatial structure of $\delta \psi_{a}^{+}(x, t)$.

We now substitute (25a) into (24). Equation (23) leads us to obtain the leading-order variation of the global-mode energy $\delta A$ as

$$
\begin{aligned}
\delta A & =\left\langle\delta \mathbf{q}_{a}^{+}, \mathbf{w}\right\rangle=\lim _{T \rightarrow \infty} \frac{1}{T} \int_{0}^{T} \int_{\Omega} \delta \psi_{a}^{+*} w+\delta \psi_{a}^{+} w^{*} d x d t \\
& =\lim _{T \rightarrow \infty} \frac{1}{T} \int_{0}^{T} \int_{\Omega} \delta \phi_{a}^{+*} e^{i \omega_{N G} t} f+\delta \phi_{a}^{+} e^{-i \omega_{N G} t} f^{*} d x d t+\int_{\Omega} \delta \phi_{a}^{+*} \phi_{N G} c+\delta \phi_{a}^{+} \phi_{N G}^{*} c^{*} d x
\end{aligned}
$$

Here, the first term in the second line of (28) implies that the spatial structure of the adjoint variable $\delta \phi_{a}^{+}$characterizes the most receptive position to the open-loop perturbation $f$, the component of which carries a spatio-temporal structure parallel to $\delta \psi_{a}^{+}\left(=\delta \phi_{a}^{+} e^{-i \omega_{N G} t}\right)$. Similarly, the second term indicates that a large drift of the amplitude can be made if $c(x)$ has a large value in the region where $\delta \phi_{a}^{+}$and $\phi_{N G}$ overlap each other. It is finally worth mentioning that the right-hand side of (28) is strictly real, consistent with the definition of $A$ as a real scalar.

If the size of the open-loop and the closed-loop perturbations are restricted to the constraints $\lim _{T \rightarrow \infty} 1 / T \int_{0}^{T} \int_{\Omega}|f(x, t)|^{2} d x d t=1$ and $\int_{\Omega}|c(x)|^{2} d x=1$ respectively, the Schwartz inequality yields

$$
\delta A \leq 2 \int_{\Omega}\left|\delta \phi_{a}^{+}(x)\right|^{2} d x+2 \int_{\Omega}\left|\phi_{N G}^{*}(x) \delta \phi_{a}^{+}(x)\right|^{2} d x .
$$


Here, the first and the second terms in the right-hand side respectively represent the maximum leading-order amplitude drifts caused by the open-loop and the closed perturbations, and the equality in (29a) satisfies if

$$
f(x, t)=\delta \phi_{a}^{+}(x) e^{-i \omega_{N G} t} \text { and } c(x)=\phi_{N G}^{*}(x) \delta \phi_{a}^{+}(x) .
$$

This suggests that, for a given energy of the open-loop perturbation, the largest amplitude drift is achieved if the perturbation is identical to the adjoint field $\psi_{a}(x, t)\left(=\delta \phi_{a}^{+}(x) e^{-i \omega_{N G} t}\right)$. Similarly, the structure of the gain $c(x)$ for the closed-loop perturbation yields the largest amplitude drift if it is identical to $\phi_{N G}^{*}(x) \delta \phi_{a}^{+}(x)$.

\subsection{Frequency}

Now, we consider the leading-order variation of the frequency $\omega_{N G}$ of a nonlinear global mode following Luchini et al. (2008). From the form of the global mode given in (10), equation (16) with $a=0$ results in the following equation:

$$
-i \omega_{N G} \psi_{N G}=L \psi_{N G}-i \gamma\left|\psi_{N G}\right|^{2} \psi_{N G} .
$$

The addition of a small perturbation then yields $\omega_{N G} \rightarrow \omega_{N G}+a \delta \omega_{N G}+O\left(a^{2}\right)$ and $\psi_{N G} \rightarrow$ $\psi_{N G}+a \delta \psi+O\left(a^{2}\right)$, resulting in the following equation at $O(a)$ :

$$
-i \delta \omega_{N G} \psi_{N G}-i \omega_{N G} \delta \psi=L \delta \psi-i 2 \gamma\left|\psi_{N G}\right|^{2} \delta \psi-i \gamma \psi_{N G}^{2} \delta \psi^{*}+w .
$$

Following Luchini et al. (2008), the key to proceed further starts from an observation that the following equation,

$$
-i \omega_{N G} \delta \psi=L \delta \psi-i 2 \gamma\left|\psi_{N G}\right|^{2} \delta \psi-i \gamma \psi_{N G}^{2} \delta \psi^{*},
$$

admits a non-trivial solution in the form of

$$
\delta \psi(x, t)=\delta \phi_{f}(x) e^{-i \omega_{N G} t},
$$

where, in the case of the CGL equation (3),

$$
\delta \phi_{f}(x)=-i \omega_{N G} \phi_{N G}(x) .
$$

It should be noted that this is a simple consequence obtained by taking $\partial / \partial t$ to equation (3). This then rearranges (31) as

$$
-i \delta \omega_{N G} \psi_{N G}+\frac{\partial \delta \psi}{\partial t}=L \delta \psi-i 2 \gamma\left|\psi_{N G}\right|^{2} \delta \psi-i \gamma \psi_{N G}^{2} \delta \psi^{*}+w .
$$

Now, similarly to the case of the amplitude, we take the complex conjugate to (33) and build the following matrix equation:

$$
-i \delta \omega_{N G} \mathbf{C} \mathbf{q}_{N G}=\mathbf{L} \delta \mathbf{q}+\mathbf{w},
$$

where

$$
\mathbf{C}=\left(\begin{array}{cc}
1 & 0 \\
0 & -1
\end{array}\right)
$$


Since $\mathbf{L} \delta \mathbf{q}=0$ has a non-trivial solution, its adjoint equation,

$$
\mathbf{L}^{+} \delta \mathbf{q}_{f}^{+}=0,
$$

with the terminal and boundary conditions

$$
\delta \mathbf{q}_{f}^{+}(x, t=\infty)=0 \text { and } \delta \mathbf{q}_{f}^{+}(x= \pm \infty, t)=0,
$$

should yield a non-trivial solution. From the first and the second row of (35a), one can easily identify that the adjoint equation is essentially given as

$$
-\frac{\partial \psi_{f}^{+}}{\partial t}=L^{+} \delta \psi_{f}^{+}+i 2 \gamma^{*}\left|\psi_{N G}\right|^{2} \delta \psi_{f}^{+}-i \gamma \psi_{N G}^{2} \delta \psi^{+*},
$$

and it admits the following form of the solution:

$$
\delta \psi_{f}^{+}(x, t)=\delta \phi_{f}^{+}(x) e^{-i \omega_{N G} t} .
$$

where $\delta \phi_{f}^{+}(x)$ is the function describing the spatial structure of $\delta \psi_{f}^{+}(x, t)$.

Once the solution of (36a) is obtained, it is straightforward to calculate the leading-order variation of the frequency $\delta \omega_{N G}$ as

$$
\begin{aligned}
\delta \omega_{N G} & =\frac{i\left\langle\mathbf{q}_{f}^{+}, \delta \mathbf{w}\right\rangle}{\left\langle\mathbf{q}_{f}^{+}, \mathbf{C} \mathbf{q}_{N G}\right\rangle}=\lim _{T \rightarrow \infty} \frac{\frac{i}{T} \int_{0}^{T} \int_{\Omega} \delta \psi_{f}^{+*} w+\delta \psi_{f}^{+} w^{*} d x d t}{\int_{\Omega} \delta \phi_{f}^{+*} \phi_{N G}-\delta \phi_{f}^{+} \phi_{N G}^{*} d x} \\
& =\lim _{T \rightarrow \infty} \frac{\frac{i}{T} \int_{0}^{T} \int_{\Omega} \delta \phi_{f}^{+*} e^{i \omega_{N G} t} f+\delta \phi_{f}^{+} e^{-i \omega_{N G} t} f^{*} d x d t}{\int_{\Omega} \delta \phi_{f}^{+*} \phi_{N G}-\delta \phi_{f}^{+} \phi_{N G}^{*} d x}+\omega_{N G} \frac{\int_{\Omega} \delta \phi_{f}^{+*} \phi_{N G} c-\delta \phi_{f}^{+} \phi_{N G}^{*} c^{*} d x}{\int_{\Omega} \delta \phi_{f}^{+*} \phi_{N G}-\delta \phi_{f}^{+} \phi_{N G}^{*} d x} .
\end{aligned}
$$

Here, it should be mentioned that both numerator and denominator in the second line of (37) are purely imaginary, indicating that the second line is purely real, consistent with the global-mode frequency defined as a real scalar. Also, similarly to the sensitivity of the amplitude given in (28), the first term in the second line of (37) suggests that the $\delta \phi_{f}^{+}$characterizes the most receptive position to the open-loop perturbation $f$ for the frequency if the component of $f$ contains a spatio-temporal structure parallel to $\delta \psi_{f}^{+}\left(=\delta \phi_{f}^{+} e^{-i \omega_{N G} t}\right)$. The second term also indicates that the frequency would experience large change if $c(x)$ contains a large value in the region where $\delta \phi_{f}^{+}$ and $\phi_{N G}$ overlap.

Similarly to the case of the amplitude, the constraints $\lim _{T \rightarrow \infty} 1 / T \int_{0}^{T} \int_{\Omega}|f(x, t)|^{2} d x d t=1$ and $\int_{\Omega}|c(x)|^{2} d x=1$ for the size of the open-loop and the closed-loop perturbations leads to the following largest drift of the frequency due to the open-loop and the closed-loop perturbations:

$$
\delta \omega_{N G} \leq \frac{2 \int_{\Omega}\left|\delta \phi_{f}^{+}(x)\right|^{2} d x+2 \omega_{N G} \int_{\Omega}\left|\phi_{N G}^{*}(x) \delta \phi_{f}^{+}(x)\right|^{2} d x}{\left|\left\langle\mathbf{q}_{f}^{+}, \mathbf{C} \mathbf{q}_{N G}\right\rangle\right|},
$$

where the first and the second terms in the right-hand side respectively indicate the maximum leading-order frequency drifts caused by the open-loop and the closed perturbations. The equality in (38a) holds if

$$
f(x, t)=\delta \phi_{f}^{+}(x) e^{-i \omega_{N G} t} \text { and } c(x)=\phi_{N G}^{*}(x) \delta \phi_{f}^{+}(x) .
$$




\subsection{Numerical method and parameters}

The sensitivity analysis formulated in the sections 3.1 and 3.2 is performed by numerically solving (3), (20), and (36). The streamwise direction is discretized using the second-order central difference on a computational domain $x \in[-100,100]$ with $N=1001$ of uniformly spaced grid points. The time integration is carried out semi-implicitly: the linear advection and diffusion terms are advanced using the second-order Crank-Nicolson method, while the rest of the terms is marched using a low-storage third-order Runge-Kutta method. A set of the reference parameters giving a soft global mode are chosen such that $k_{0}=0.5, \omega_{k k}=-i, \omega_{0}^{\max }=0.4+0.5 i, \omega_{0 x x}=$ $-0.0004-0.002 i$, and $\gamma=-i$. Two parameters $k_{0, i}$ and $\omega_{0, i}^{\max }$ respectively controlling advection velocity and bifurcation are then varied: $k_{0, i}=0 \sim-0.9$ and $\omega_{0, i}^{\max }=0.1 \sim 0.5$. The results discussed in the present study have been tested for a few different sets of parameters and show qualitatively no difference.

\section{Results \& Discussion}

Thus far, we have formulated the leading-order variations of the amplitude and the frequency of a nonlinear global mode as summarized (28) and (37) respectively. Based on these formulations, we discuss structural sensitivity of a given nonlinear global mode. For the sensitivity to open-loop perturbation, we will visualize $\left|\delta \phi_{a}^{+}(x)\right|$ and $\left|\delta \phi_{f}^{+}(x) /\left\langle\mathbf{q}_{f}^{+}, \mathbf{C} \mathbf{q}_{N G}\right\rangle\right|$ which respectively present the leading-order variation of the amplitude and the frequency. On the other hand, for the sensitivity to closed-loop perturbation, $\left|\phi_{N G}^{*}(x) \delta \phi_{a}^{+}(x)\right|$ and $\left|\phi_{N G}^{*}(x) \delta \phi_{f}^{+}(x) /\left\langle\mathbf{q}_{f}^{+}, \mathbf{C} \mathbf{q}_{N G}\right\rangle\right|$ will be shown.

\subsection{Soft global mode}

The structural sensitivities of the amplitude and the frequency of a soft nonlinear global mode are plotted in figure 4. They all exhibit appreciable values in the region of $x_{s a}<x<$ $x_{a s}$ in which the medium is locally linearly unstable, indicating that the entire region of the local linear (absolute) instability $\left(\operatorname{Im}\left(\Omega_{l}\right)>0\right)$ plays a crucial role in characterizing the most receptive region of soft global mode. It is particularly worth noting that the sensitivity of the frequency to the closed-loop perturbation shows a relatively sharp peak at $x=x_{s}^{n l}$ (figure $4 d$ ), indicating that the frequency is highly sensitive to a small noise of the intrinsic parameters at this location. This is consistent with the previous WKBJ analysis by Pier and Huerre (1996) and Pier et al. (2001) in which the frequency of soft global mode is determined by a combination of the intrinsic parameters of the CGL equation (3) through the criterion (13). Finally, it should be mentioned that the spatial structure of the sensitivities of the amplitude exhibits three local maxima (figures $4 a$ and $b$ ), contrary to that of the frequency (figures $4 c$ and $d$ ). The two of the peaks in the sensitivities of the amplitude appear at the locations not so far from $x=x^{s a}$ and $x=x^{a s}$ respectively, indicating that perturbing around these locations would significantly change the amplitude of the global mode while it does not yield a significant change of the frequency. It is difficult to address the precise reason why three peaks appear in that case. However, it is worth noting that the two peaks are seen to appear around the locations where the amplitude of the soft global mode is small: i.e. $x=x_{s a}$ and $x=x_{a s}$. At these locations, the homogeneous part of the tangent equation (19) would be locally marginally stable, indicating that forcing around this location may yield a large response although this is merely a speculation.

The effect of the bifurcation control parameter $\omega_{0, i}^{\max }$ is examined as reported in figure 5 . Except for the smallest $\omega_{0, i}^{\max }(=0.1)$, the main features of the sensitivities of the amplitude themselves are not significantly affected on changing $\omega_{0, i}^{\max }$ (figures $5 a$ and $b$ ): for example, both $\left|\delta \phi_{a}^{+}\right|$ 

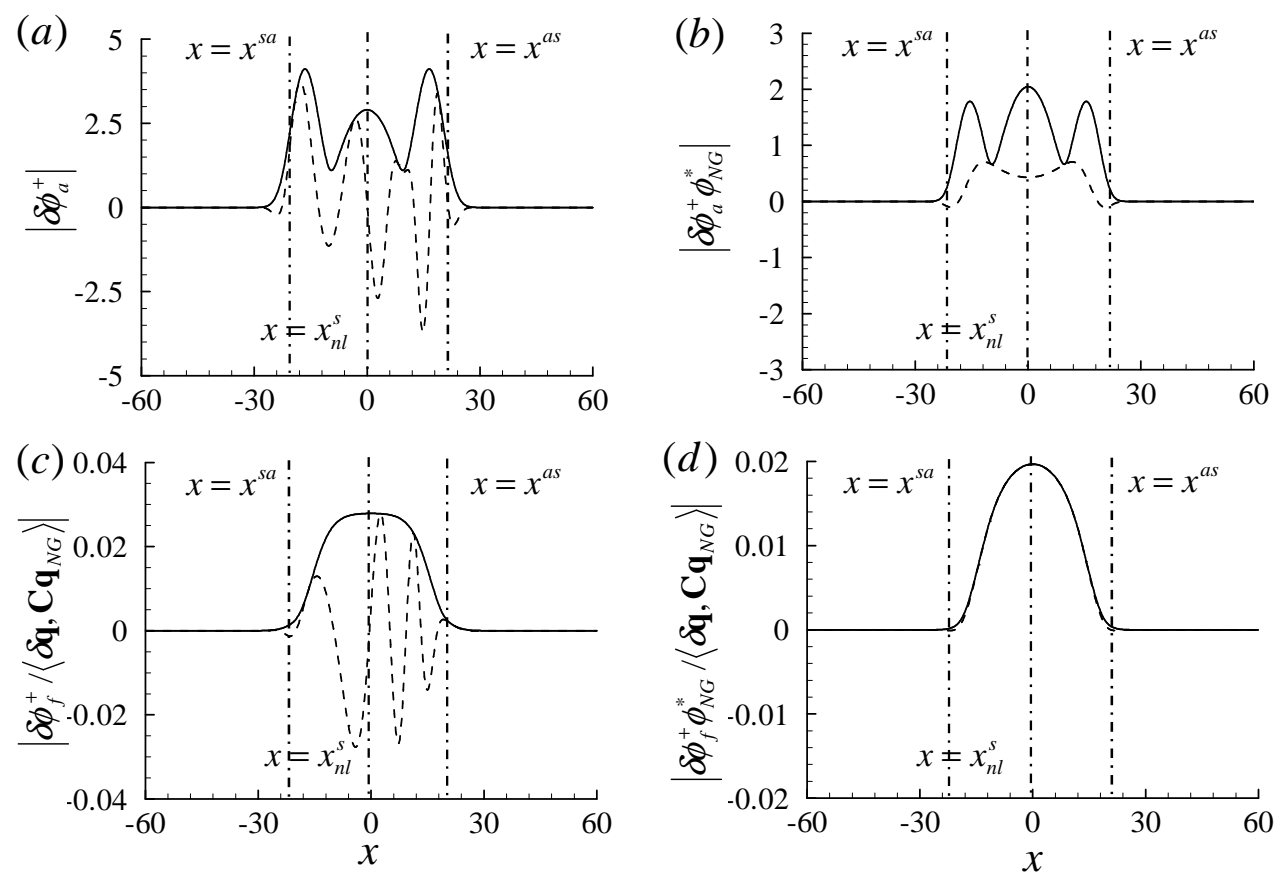

Figure 4: Structural sensitivities of $(a, b)$ the amplitude and $(c, d)$ the frequency of soft nonlinear global mode to (a,c) open-loop and $(b, d)$ closed-loop perturbations. The vertical dash-dotted lines from left to right indicate $x=x^{s a}, x=x_{n l}^{s}$, and $x=x^{s a}$, respectively (see also section 2.2.1 for the details on these locations). Here, the curves are: $-\frac{n l}{\text {, }},|\cdot|$; ,$---- \operatorname{Re}(\cdot)$. 

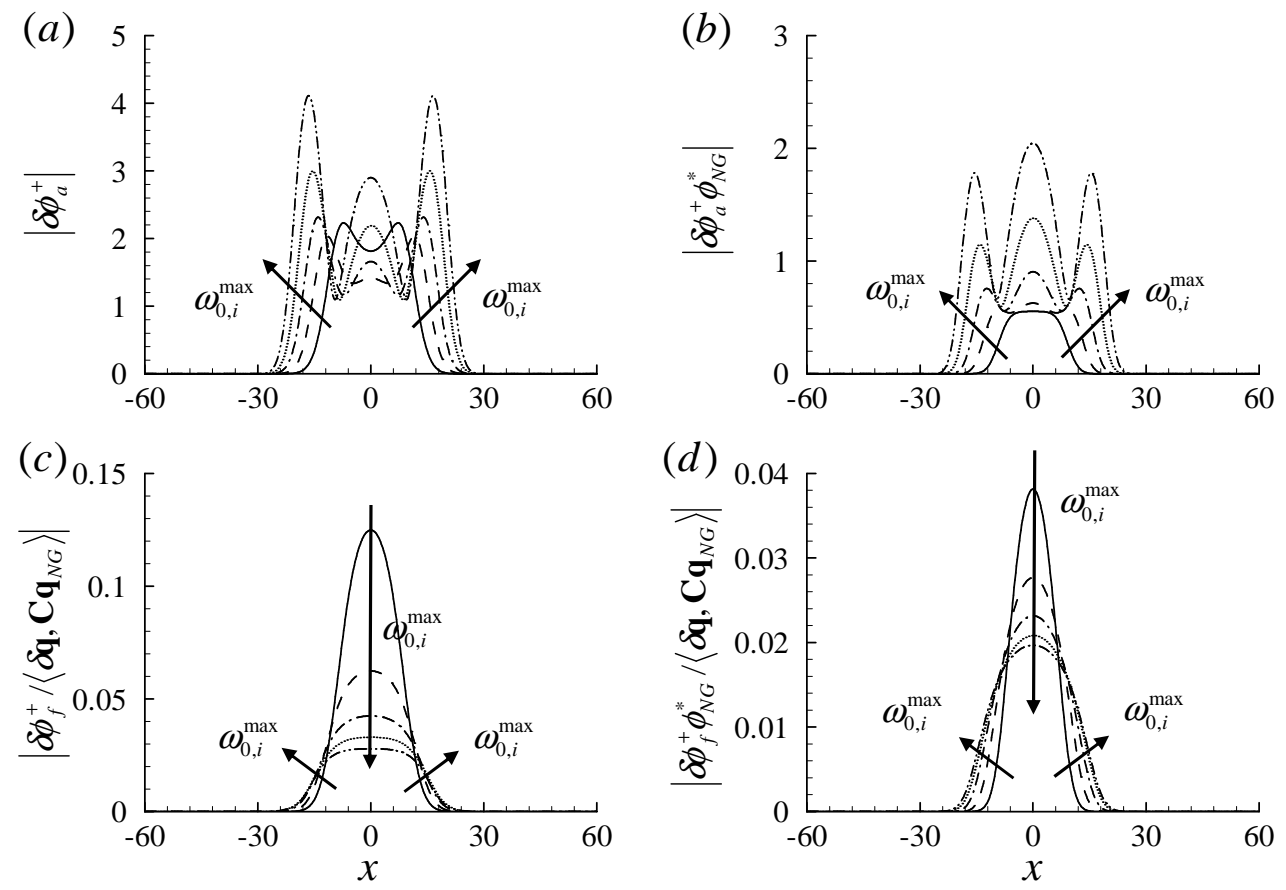

Figure 5: Dependence of structural sensitivities of $(a, b)$ the amplitude and $(c, d)$ the frequency of soft nonlinear global mode to $(a, c)$ open-loop and $(b, d)$ closed-loop perturbations on the maximum absolute growth rate $\omega_{0, i}^{\max }$ : $\omega_{0, i}^{\max }=0.1 ;----, \omega_{0, i}^{\max }=0.2 ;-\cdot-\cdot-, \omega_{0, i}^{\max }=0.3 ; \cdots \cdots \cdots, \omega_{0, i}^{\max }=0.4-\cdots-\cdots-, \omega_{0, i}^{\max }=0.5$. 

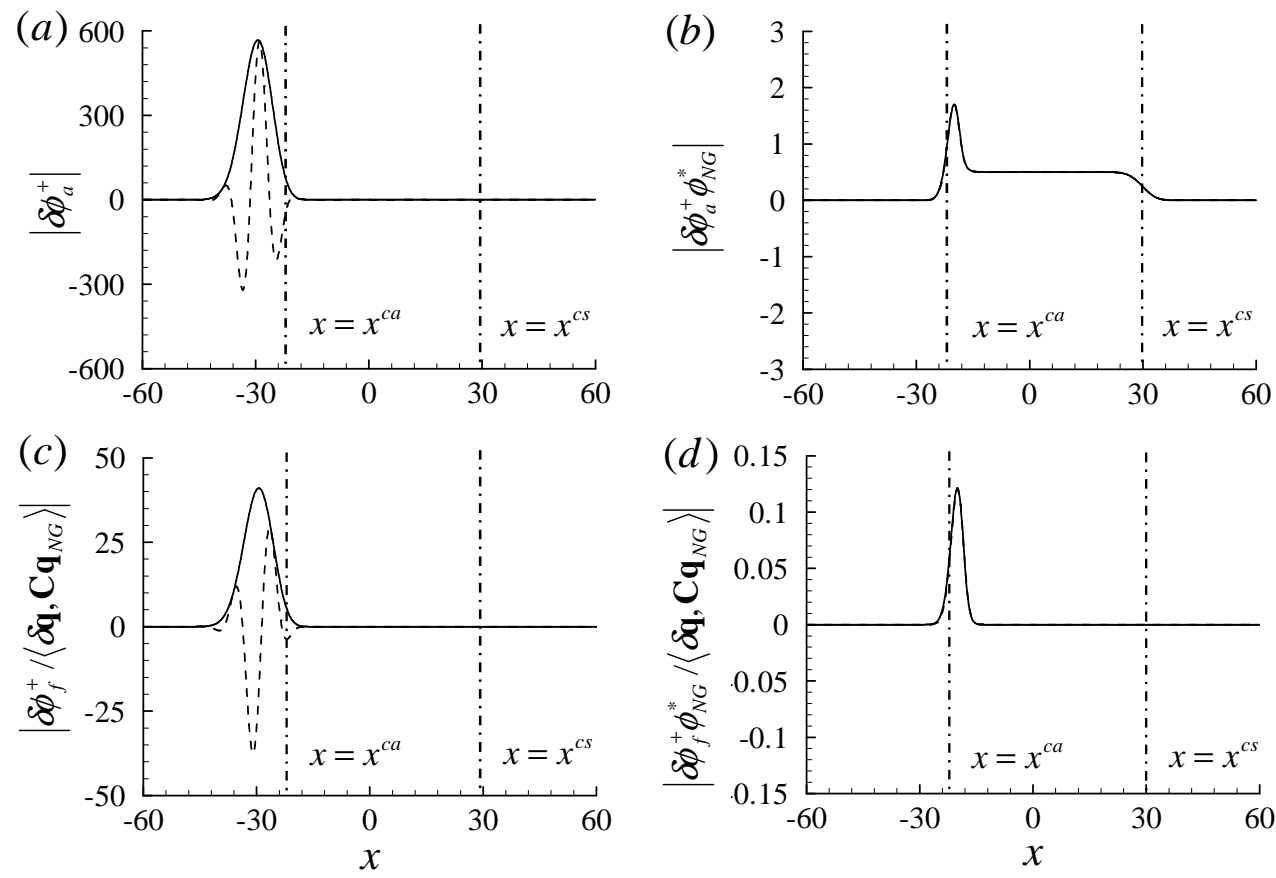

Figure 6: Structural sensitivities of $(a, b)$ the amplitude and $(c, d)$ the frequency of steep nonlinear global mode to $(a, c)$ open-loop and $(b, d)$ closed-loop perturbations. The vertical dash-dotted lines from left to right indicate $x=x^{c a}$ and $x=x^{c s}$, respectively (see also section 2.2 .2 for the details on these locations). The curves are:,$--|\cdot| ;----$, $\operatorname{Re}(\cdot)$.

and $\mid \delta \phi_{a}^{+} \delta \phi_{N G}^{*}$ maintain the three local maxima with respect to the change of $\omega_{0, i}^{\max }$. Even for $\omega_{0, i}^{\max }=0.1$, the peak at $x=x_{n l}^{s}$ simply appears to be hidden by the two peaks near $x=x^{s a}$ and $x=x^{a s}$ approached too close to each other; this probably explains why the overall value of $\left|\delta \phi_{a}^{+}\right|$ for $\omega_{0, i}^{\max }=0.1$ is larger than that for $\omega_{0, i}^{\max }=0.1$. However, on changing $\omega_{0, i}^{\max }$, the sensitivities of the amplitude also exhibit some subtle changes. First, the support of the sensitivities (the interval in which the sensitivities are non-negligible) becomes widen on increasing $\omega_{0, i}^{\max }$ probably due to widening of the region of the local linear (absolute) instability $\left(\operatorname{Im}\left(\Omega_{l}\right)>0\right)$. Also, the overall value of the amplitude sensitivities exhibits augmentation on increasing $\omega_{0, i}^{\max }$, indicating that the amplitude of the soft global mode is more easily perturbed as $\omega_{0, i}^{\max }$ increases. In contrast, the sensitivities of the frequency are seen to behave a little differently (figures $5 c$ and $d$ ). While the spatial structure of the frequency sensitivities (i.e. the appearance of a single peak at $x=x_{n l}^{s}$ ) is maintained on changing $\omega_{0, i}^{\max }$ as that of the amplitude, the peak value at $x=x_{n l}^{s}$ in the sensitivities of the frequency to both open-loop and closed-loop perturbations rapidly decreases with the increase of $\omega_{0, i}^{\max }$.

\subsection{Steep global mode}

Figure 6 shows the structural sensitivities of the amplitude and the frequency of a steep nonlinear global mode. In contrast to the case of soft global mode (figure 4), they all show large values upstream. It is evident that this is a consequence of spatial causality resulting from 

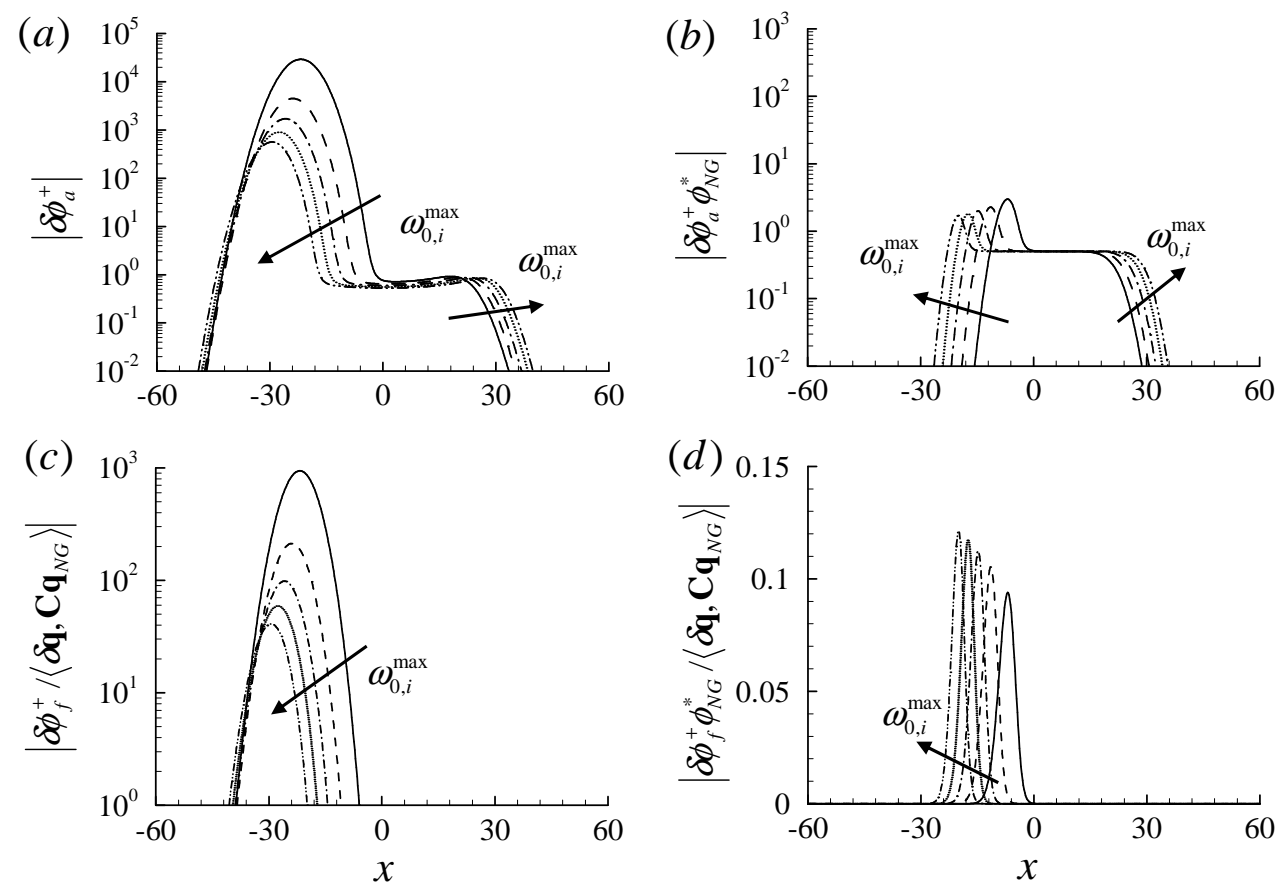

Figure 7: Dependence of structural sensitivities of $(a, b)$ the amplitude and $(c, d)$ the frequency of steep nonlinear global mode to $(a, c)$ open-loop and $(b, d)$ closed-loop perturbations on the maximum absolute growth rate $\omega_{0, i}^{\max }$ : $\omega_{0, i}^{\max }=0.1 ;----, \omega_{0, i}^{\max }=0.2 ;-\cdot-\cdot-, \omega_{0, i}^{\max }=0.3 ; \cdots \cdots \cdots, \omega_{0, i}^{\max }=0.4-\cdots-\cdots-, \omega_{0, i}^{\max }=0.5$.

downstream advection $\left(U_{r}=0.9\right)$. Both the sensitivities of the amplitude and the frequency to open-loop perturbation (figures $6 a$ and $b$ ) clearly reveal large values upstream of $x=x^{c a}$ where the steep global mode shows negligibly small values (see also figure 2). The large sensitivities in this region imply that the global mode is most receptive to an open-loop perturbation provided in this region and this is consistent with Pier (2003) where an open-loop forcing provided at the upstream convectively unstable region yields a large response. On the other hand, the sensitivities of the amplitude and the frequency to a closed-loop perturbation (figures $6 c$ and $d$ ) exhibit highly localized peaks near $x=x^{c a}$ where the stationary front is located (see figure 2). This suggests that the intrinsic parameters such as $\omega_{0}(x)$ have crucial significance at this location, demonstrating the role of the front as wavemaker of steep global mode found by the previous WKBJ analysis (Pier et al., 1998, 2001) using a sensitivity analysis. Finally, it should be mentioned that the sensitivity of the amplitude to closed-loop perturbation also shows non-negligible values in the region of $x^{c a}<x<x^{c s}$, indicating that perturbing the location where the nonlinear spatial branch $\left(k_{n l}^{+}\right)$is dominant also yields some changes in the amplitude of steep global mode.

The effect of the bifurcation control parameter $\omega_{0, i}^{\max }$ is also studied as shown in figure 7 . On increasing $\omega_{0, i}^{\max }$, the support of the sensitivities of the amplitude of steep global mode to open-loop and closed-loop perturbations becomes wider (figures $7 a$ and $b$ ) similarly to those of soft global mode (figures $5 a$ and $b$ ). However, contrary to the case of soft global mode, the peak values of the amplitude sensitivities of steep global mode were found to decay with the increase of $\omega_{0, i}^{\max }$. It is worth pointing out that the same feature was also observed in the steep 

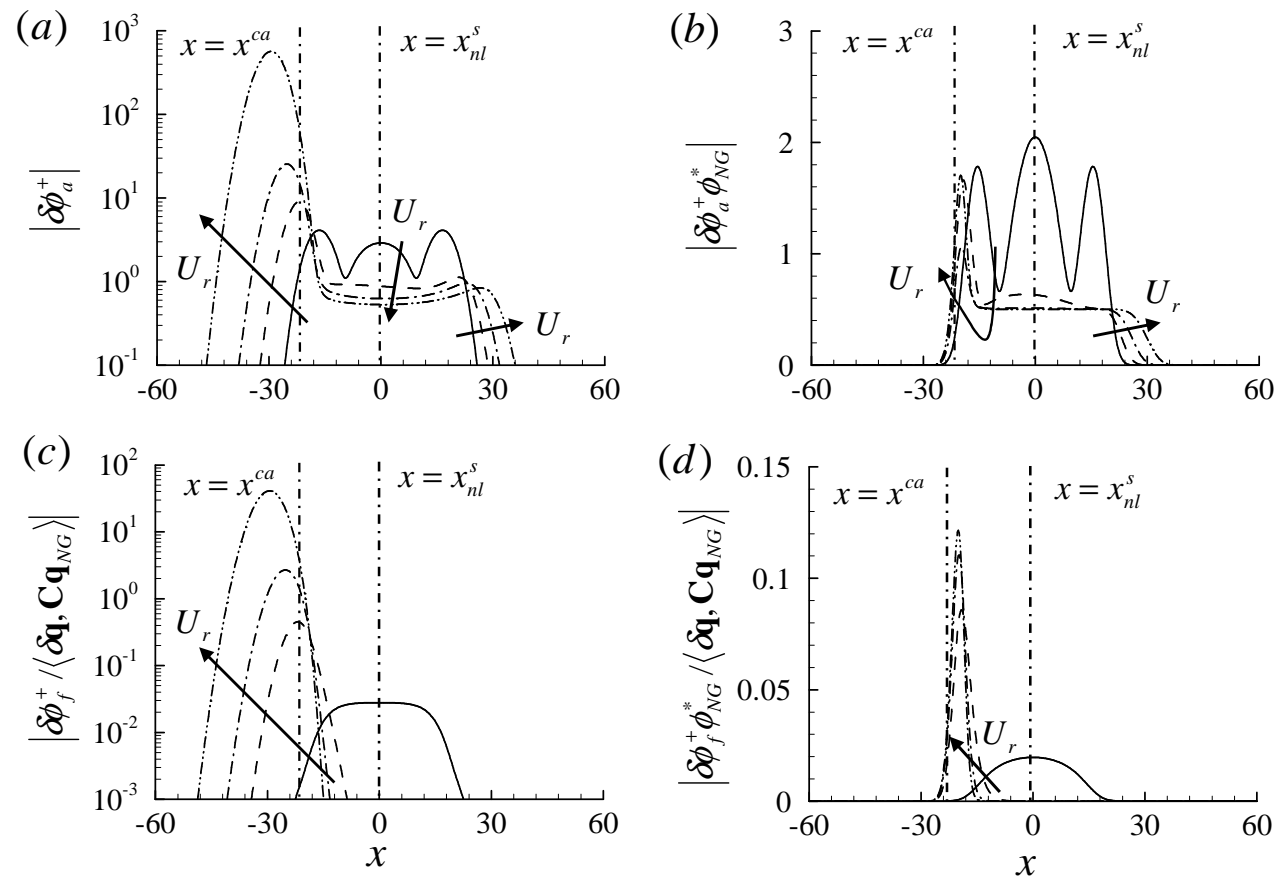

Figure 8: Dependence of structural sensitivities of $(a, b)$ the amplitude and $(c, d)$ the frequency to $(a, c)$ open-loop and $(b, d)$ closed-loop perturbation on the advection velocity $\left(U_{r}=-\omega_{k k} k_{0}\right):-, U_{r}=0 ;----, U_{r}=0.3 ;-\cdot-\cdot-$, $U_{r}=0.6 ;-\cdots-\cdots, U_{r}=0.9$.

global mode in supercritical real Ginzburg-Landau equation, suggesting that this may be a robust feature at least for the family of steep global modes in Ginzburg-Landau equation. However, for the sensitivity of the frequency, such a decrease in its peak value is only observed in that to openloop perturbation (figure $7 c$ ). In fact, the sensitivity of the frequency to closed-loop perturbation appears to rather slightly increase with $\omega_{0, i}^{\max }$ (note that the vertical axis in figures $7 a, b$, and $c$ is in logarithmic scale while that in figure $7 d$ is in algebraic scale). This implies that the role of the stationary front in determining frequency is not significantly disturbed on increasing $\omega_{0, i}^{\max }$.

\subsection{Transition of the structural sensitivity from soft to steep global modes}

The structural change in the sensitivities on increasing the advection velocity $\left(U_{r}=-\omega_{k k} k_{0}\right)$ is shown in figure 8 . In the absence of the advection velocity $\left(U_{r}=0\right)$, all the structural sensitivities exhibit appreciable value in the region of local linear absolute instability as already discussed (see also figure 4). Although the presence of the advection velocity does not change the region of the local absolute instability, the structural sensitivities are significantly changed. In the case of open-loop perturbation, the sensitivities of both the amplitude and the frequency in the region of local linear absolute instability decay with the increase of the advection velocity (figures $8 a$ and $c$ ). Furthermore, the region exhibiting the largest sensitivity gradually moves upstream on increasing the advection velocity. More importantly, the maximum value in the sensitivities to open-loop perturbation exponentially increases (note that the scale of the vertical axis in figures $8 a$ and $c$ is logarithmic). It should be mentioned that this is presumably a consequence of the in- 

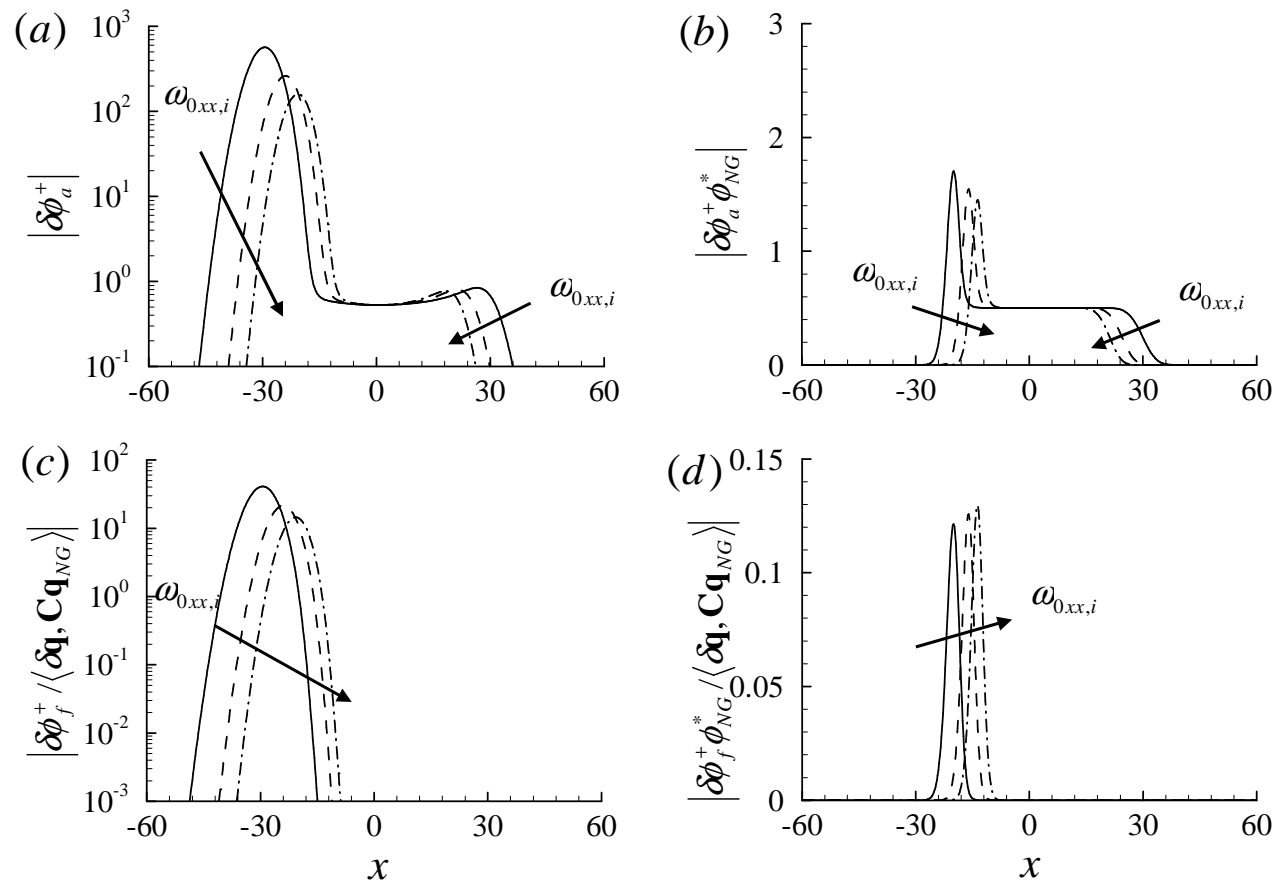

Figure 9: Dependence of structural sensitivities of $(a, b)$ the amplitude and $(c, d)$ the frequency to $(a, c)$ open-loop and

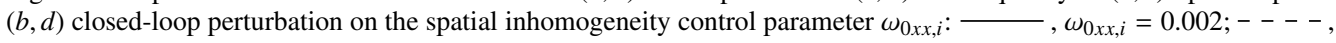
$\omega_{0 x x, i}=0.003 ;----, \omega_{0 x x, i}=0.004$.

creased convective non-normality of the linearized equations (19) and (34) (Cossu and Chomaz, 1997). In contrast, the behavior of the structural sensitivities to closed-loop perturbation (figures $8 b$ and $d$ ) is quite different from that to open-loop perturbation. For example, the upstream migration of the peak location in the sensitivities appears only in a small range of the increase of the advection velocity (roughly $0 \leq U_{r} \leq 0.3$ ). In fact, the peak location does not move further upstream even if the advection velocity is much more increased from $U_{r}=0.3$, and its location firmly settles into near $x=x^{c a}$ where the stationary front is located. More interestingly, the increase of the peak value with the advection velocity is not as significant as that observed in the case of open-loop perturbation. This suggests that the role of the stationary front as a wavemaker appears to be a very robust feature at least for the steep global mode when the advection is sufficiently large. Finally, it should be mentioned that examination of the sensitivities for small values of advection $\left(0<U_{r}<0.3\right)$ reveals that the peak locations in all the sensitivities are found to smoothly move upstream although the structural change of the amplitude sensitivity is not trivial. However, this feature is limited only for small advection where transition from soft to steep mode takes place.

\subsection{Role of spatial inhomogeneity on sensitivities of steep global mode}

Role of spatial inhomogeneity is reported in figure 9 where the sensitivities on decreasing $\omega_{0 x x, i}$ are plotted. Here, we note that only the steep global mode is shown as it is the one often observed in real flows. Since an increase of $\omega_{0 x x, i}$ reduces the size of local convective and 
absolute instabilities, it is seen that both sensitivities of the amplitude and the frequency to openloop perturbations generally become smaller move downstream on increasing $\omega_{0 x x, i}$ (figures $9 a$ and $c$ ). It is worth mentioning that this behaviour may be related to the shrunken convectively unstable region by the decrease of $\omega_{0 x x, i}$, which probably acts as perturbation amplifier. In contrast, the sensitivities to closed-loop perturbations do not experience such a drastic change. Their peak locations move downstream as $x^{c a}$ does with a decrease of $\omega_{0 x x, i}$, but their values do not significantly change (figures $9 b$ and $d$ ). However, this is not surprising, given the fact that the closed-loop perturbation is effective at the location where the global-mode amplitude is large enough.

\subsection{Comparison with weakly nonlinear analysis}

Finally, we perform a weakly nonlinear analysis of (3) to compare it with the sensitivity analysis of fully nonlinear global modes shown so far. The weakly nonlinear analysis here exactly follows the one given in Chomaz (2005), thus only a brief summary of the analysis is given (for the further details, the reader may refer to Chomaz (2005)). We start by carrying out a global linear stability analysis of (3), leading to the eigenvalue problem (7). Equation (7) admits exact analytic solution (Cossu and Chomaz, 1997; Chomaz, 2005), giving the most unstable (or least stable) eigenvalue

$$
\omega_{L G}=\omega_{0}^{\max }-i \frac{3}{2}\left|\frac{\omega_{0 x x} \omega_{k k}}{2}\right|^{1 / 2} e^{i\left(\arg \left(i \omega_{0 x x}\right)+\arg \left(i / 2 \omega_{k k}\right)\right) / 2}
$$

with the corresponding eigenfunction

$$
\delta \phi_{L G}(x)=\delta \phi_{0} e^{i k_{0} x-\chi^{2} x^{2} / 2}
$$

Here, $\chi=\left|\omega_{0 x x} \omega_{k k} / 8\right|^{1 / 4} e^{i\left(\arg \left(i \omega_{0 x x}\right)+\arg \left(i / 2 \omega_{k k}\right)\right) / 4}$ and $\delta \phi_{0}$ is a constant giving $\int_{\Omega}\left|\delta \phi_{L G}(x)\right|^{2} d x=1$.

We now recast equation (16) with $a \ll 1$. We consider the critical value of the bifurcation control parameter $\omega_{0, i}^{\max }=\omega_{0, i}^{c}$ resulting in neutrally stable global frequency $\omega_{L G}^{c}$ (i.e. $\operatorname{Im}\left(\omega_{L G}^{c}\right)=$ 0 . We then set $\omega_{0, i}^{\max }=\omega_{0, i}^{c}+a \Delta \omega_{0, i}^{\max }$ where $\Delta \omega_{0, i}^{\max }$ characterizes the departure from the onset of the linear global instability. If $f(x, t)=a^{1 / 2} f_{0}(x, T) e^{-i \omega_{L G}^{c} t}$ is assumed, the standard multiplescale analysis then allows the solution of (16) at $O\left(a^{1 / 2}\right)$ to take $\psi(x, t)=A(T) \delta \psi_{L G} e^{-i \omega_{L G}^{c} t}$ with $T=a t$, resulting in the following amplitude equation at $O\left(a^{3 / 2}\right)$ :

$$
\frac{d A}{d T}=\Delta \omega_{0, i}^{\max } A-i \gamma \frac{\left[\delta \phi_{L G}^{+},\left|\delta \phi_{L G}\right|^{2} \delta \phi_{L G}\right]}{\left[\delta \phi_{L G}^{+}, \delta \phi_{L G}\right]}|A|^{2} A+\frac{\left[\delta \phi_{L G}^{+}, f_{0}(x, T)\right]}{\left[\delta \phi_{L G}^{+}, \delta \phi_{L G}\right]}+\frac{\left[\delta \phi_{L G}^{+}, c(x) \delta \phi_{L G}\right]}{\left[\delta \phi_{L G}^{+}, \delta \phi_{L G}\right]} A
$$

where $[g, h]=\int_{\Omega} g^{*} h d x$ denotes the standard inner product and $\phi_{L G}^{+}$is the corresponding adjoint variable given as

$$
\delta \phi_{L G}^{+}(x)=\delta \phi_{0}^{+} e^{i k_{0}^{*} x-\chi^{2} x^{2} / 2},
$$

where $\delta \phi_{0}^{+}$is normalization constant giving $\int_{\Omega}\left|\delta \phi_{L G}^{+}(x)\right|^{2} d x=1$.

Equation (40a) now becomes the equation governing dynamics of the global mode in the presence of driven perturbations. In particular, the third term in the right-hand side of (40a) represents the role of the open-loop perturbation. It suggests that the the spatial structure of $\delta \phi_{L G}^{+}$ characterizes the most receptive position to the open-loop perturbation $f_{0}$. Similarly, the last term in the right-hand side of (40a) represents the role of the closed-loop perturbation and indicates that that the region where $\delta \phi_{L G}^{+}$and $\delta \phi_{L G}$ visualizes the place that the feedback gain $c(x)$ in the closed-loop perturbation acts most efficiently. 

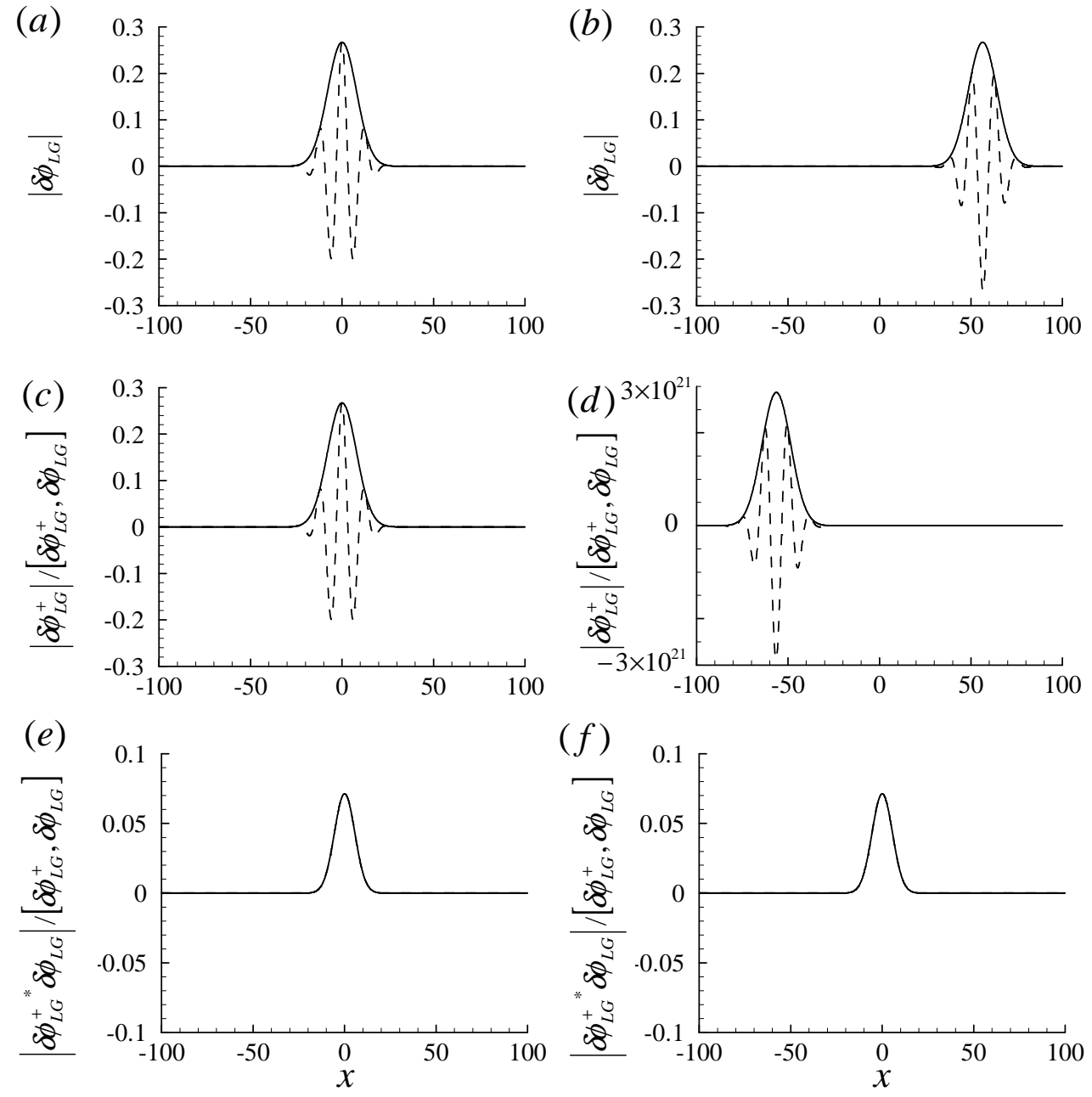

Figure 10: $(a, b)$ Linear global mode and its structural sensitivities to $(c, d)$ open-loop and $(e, f)$ closed-loop perturbations for $(a, c, g) U_{r}=0$ and $(b, d, h) U_{r}=0.9$. Here, the dashed curves indicate the real part. 
Figure 10 reports the linear global mode and the corresponding structural sensitivities to open-loop and closed-loop perturbations for the advection velocities $U_{r}=0$ and $U_{r}=0.9$, which respectively results in soft and steep nonlinear global modes in the fully nonlinear regime (i.e. for sufficiently large $\left.\omega_{0, i}^{\max }\right)$. For $U_{r}=0\left(k_{0, i}=0\right)$, both the regular and the adjoint global modes are located around $x=0$ due to the absence of the advection (figures $10 a$ and $c$ ). In fact, the adjoint global mode is identical to the regular one (i.e. $\delta \phi_{L G}^{+}=\delta \phi_{L G}$ ), indicating that the linear operator $L$ is self-adjoint. Therefore, $\left[\delta \phi_{L G}^{+}, \delta \phi_{L G}\right]=1$, and the sensitivity to closedloop perturbation $\delta \phi_{L G}^{+}{ }^{*} \delta \phi_{L G} /\left[\delta \phi_{L G}^{+}, \delta \phi_{L G}\right]$ is obviously found to be located near $x=0$ (figure $10 e)$. On the other hand, for $U_{r}=0.9\left(k_{0, i}=-0.9\right)$, the region that the support of the regular global mode appears far downstream from $x=0$ due to the advection (figure $10 \mathrm{~b}$ ), whereas that of the adjoint mode appears far upstream from $x=0$ (figure $10 d$ ). Although the sensitivity to closed-loop perturbation $\delta \phi_{L G}^{+}{ }^{*} \delta \phi_{L G} /\left[\delta \phi_{L G}^{+}, \delta \phi_{L G}\right]$ is identical to that for $U_{r}=0$ due to $\delta \phi_{L G}^{+}(x)=$ $\delta \phi_{L G}^{*}(-x)$ (see (39b) and (40b)), a very small value of $\left[\delta \phi_{L G}^{+}, \delta \phi_{L G}\right]\left(=9.3 \times 10^{-23}\right)$ is found, indicating that the linear operator $L$ in this case is highly non-normal.

Let us now compare the present sensitivities obtained using the weakly nonlinear analysis with that using the fully nonlinear analysis given in the sections 4.1 and 4.2. For $U_{r}=0$ which gives a soft global mode with sufficiently large $\omega_{0, i}^{\max }$, the spatial structures of the sensitivities from the weakly nonlinear analysis (figures $10 c$, and $e$ ) compare reasonably well with those from fully nonlinear analysis (figure 5 particularly with $\omega_{0, i}^{\max }=0.1$ ) in the sense that the sensitivities all show quite large values at $x=0$. Here, it should be noted that the first Landau constant in (40a) is reasonably large in this case $\left(i \gamma\left[\delta \phi_{L G}^{+},\left|\delta \phi_{L G}\right|^{2} \delta \phi_{L G}\right] /\left[\delta \phi_{L G}^{+}, \delta \phi_{L G}\right]=0.05\right)$. In this respect, this reasonable agreement may not be so surprising as discussed in Chomaz (2005). Furthermore, it should also be mentioned that the location $x=0$ at which the sensitivities from the weakly and the fully nonlinear analyses show large values is the place where the frequency of both the linear and the nonlinear global modes is determined according to the WKBJ theories (see (1) and (13)). In sharp contrast, for $U_{r}=0.9$, the spatial structures of the sensitivities from the weakly nonlinear analysis (figures $10 d$, and $f$ ) are very different from those from fully nonlinear analysis (figures 6 and 7). The sensitivities obtained from weakly nonlinear analysis clearly fail to capture the nonlinear wavemaker region $x=x^{c a}$ of steep global mode, suggesting that the sensitivities from the weakly nonlinear analysis in this case is not reliable. It should be mentioned that, in this case, the first landau constant is extremely small $\left(i \gamma\left[\delta \phi_{L G}^{+},\left|\delta \phi_{L G}\right|^{2} \delta \phi_{L G}\right] /\left[\delta \phi_{L G}^{+}, \delta \phi_{L G}\right]=\right.$ $4.5 \times 10^{-35}$ ), implying that the saturation amplitude of $A$ reaches $O(1)$ only with a very small $\Delta \omega_{0, i}^{\max }$. This suggests that the size of $\Delta \omega_{0, i}^{\max }$ that the weakly nonlinear theory is valid is expected to be extremely small (Chomaz, 2005), hence the result here would not be so surprising.

\section{Concluding Remarks}

In the present study, we have investigated structural sensitivities of nonlinear global modes in a complex Ginzburg-Landau equation. The main findings in the present study are summarized as follows:

1. Sensitivities of the amplitude and the frequency of a nonlinear global mode to open-loop and closed-loop perturbations are formulated following Hwang and Choi (2008) and Luchini et al. (2008), and they are applied to two types of nonlinear global modes arising in the complex Ginzburg-Landau equation: soft and steep global modes.

2. Both the amplitude and the frequency of soft global mode are shown to be very sensitive to open-loop and closed-loop perturbations in the region of local (absolute) instability. In 
particular, the sensitivity of the frequency to closed-loop perturbation exhibits its maximum at $x=x_{n l}^{s}$ at which the frequency of soft global mode was shown to be determined in the previous WKBJ analysis (Pier and Huerre, 1996).

3. The amplitude and the frequency of steep global mode are found to be fairly sensitive to open-loop perturbations provided far upstream. To close-loop perturbation, they respond very sensitively near $x=x^{c a}$ where the stationary front is located. This is also consistent with the previous WKBJ analysis (Pier et al., 1998, 2001) where the stationary front at this location is found to act as a wavemaker of soft global mode.

4. Finally, an weakly nonlinear analysis is performed to compare with the present results obtained with fully nonlinear global modes. For soft global mode, the weakly nonlinear analysis reasonably predicts the sensitive region to the perturbation found in the present analysis. However, for steep global mode, it clearly fails to capture the sensitive region found here (e.g. the location of the stationary front). It is shown that this failure essentially originates from the non-normality caused by the advection (Cossu and Chomaz, 1997), consistent with the discussion given in Chomaz (2005).

Probably, the most important finding of the present study is that the sensitivity analysis of steep nonlinear global mode confirms the role of the stationary front, consistent with the previous WKBJ analysis. Together with the local analysis, the present analysis with the toy model also explains why the frequency selection criterion (2) has been successful in predicting the frequency of the nonlinear global modes in a number of canonical flows (e.g. Pier, 2002; Lesshafft et al., 2006; Gallaire et al., 2006). The sensitivity analysis provides an example that the weakly nonlinear analysis is not indeed valid for steep global modes particularly when the base flow is weakly non-parallel and the bifurcation control parameter such as the Reynolds number is far above the onset of the primary global mode. This would also provide a warning for the approaches which simply extend the weakly nonlinear analysis to the fully nonlinear regime without careful examination of the role of the convective non-normality.

It is also worth mentioning that the form of the perturbations in the present study study is general, thus one may think of them as an open-loop and a closed-loop actuation for control. In other words, the sensitivities presented here are also a measure of 'controllability' of an openloop and a closed-loop actuation although its precise definition differs from that given in the control literatures where it is rather defined in term of 'reachability' in phase space. Therefore, the findings in the present suggest that an actuator for control of the given nonlinear global mode should be located in the region where the relevant sensitivity exhibits a large value large sensitivities for their effective performance.

Finally, one should realize that the present approach particularly for the sensitivity of the amplitude is simply a part of optimal control theory which is often aimed at calculating the leading-order variation of a given objective functional (see e.g. the early work by Abergel and Temam, 1990). Therefore, this implies that it may be extended to the system yielding chaotic behavior. However, it should be mentioned that, for the chaotic system, the present approach becomes ill-posed because the tangent equation such as (20) would not provide a converging solution due to the diverging Lyapunov exponent. This issue has been addressed in the community working on the uncertainty quantification (see e.g. Wang, 2012) and is an essential step to take particularly if one is to tackle turbulent flows using this type of approaches. 


\section{Acknowledgements}

This paper is dedicated to Prof. Patrick Huerre in honor of the Euromech Colloquium on 'Trends in Open Shear Flow Instability' held for him in July, 2013. My early research was highly influenced by his seminal early work, which made me take a long journey to Laboratoire d'Hydrodynamique at École Polytechnique (LadHyX) in 2008. Since then, I have personally and scientifically been privileged from his invaluable advices and support, and I would like to express my deepest gratitude for this.

This work was carried out by spending a significant amount of time during my postdoctoral research at Cambridge. I would like to gratefully acknowledge my advisor, Prof. Tim Pedley, for his encouragement to perform this work. This work was partially supported by the European Commission through a Marie Curie fellowship.

\section{References}

Abergel, F., Temam, R., 1990. On some control problems in fluid mechanics. Theo. Comp. Fluid Dyn. 1, $303-325$.

Bers, A., 1983. Space-time evolution of plasma instabilities - absolute and convective, in: Rosenbluth, M.N., Sagdeev, R.Z. (Eds.), Handbook of Plasma Physics. North-Holland. volume 1, pp. 451-517.

Briggs, R.J., 1964. Electron-Stream Interaction with Plasmas. MIT Press, Cambridge, Mass.

Chomaz, J.M., 1992. Absolute and convective instability in non linear systems. Phys. Rev. Lett. 69, 1931-1934.

Chomaz, J.M., 2003. Fully nonlinear dynamiques of parallel wakes. J. Fluid Mech. 485, 57-75.

Chomaz, J.M., 2005. Global instabilities in spatially developing flows: Nonnormality and nonlinearity. Annu. Rev. Fluid Mech. 37, 357-392.

Chomaz, J.M., Huerre, P., Redekopp, L.G., 1988. Bifurcations to local and global modes in spatially developing flows. Phys. Rev. Lett. 60, 25-28.

Chomaz, J.M., Huerre, P., Redekopp, L.G., 1991. A frequency selection criterion in spatially developing flows. Stud. Appl. Math. 84, 119-144.

Cossu, C., Chomaz, J.M., 1997. Global measures of local convective instabilities. Phys. Rev. Lett. 78, 4387-90.

Couairon, A., Chomaz, J.M., 1996. Global instability in fully nonlinear systems. Phys. Rev. Lett 77, 4015-4018.

Couairon, A., Chomaz, J.M., 1997a. Absolute and convective instabilities, front velocities and global modes in nonlinear systems. Physica D 108, 236-276.

Couairon, A., Chomaz, J.M., 1997b. Pattern selection in the presence of a cross flow. Phys. Rev. Lett 79, $2666-2669$.

Couairon, A., Chomaz, J.M., 1999a. Fully nonlinear global modes in slowly varying flows. Phys. Fluids 11, 3688-3703.

Couairon, A., Chomaz, J.M., 1999b. Primary and secondary nonlinear global instability. Physica D 132, 428-456.

Delbende, I., Chomaz, J.M., 1998. Nonlinear convective/absolute instabilities of parallel two-dimensional wakes. Physics of Fluids 10, 2724-2736.

Gallaire, F., Ruith, M., Meiburg, E., Chomaz, J.M., Huerre., P., 2006. Spiral vortex breakdown as a global mode. J. Fluid Mech. 549, 67-93.

Giannetti, F., Luchini, F., 2007. Structural sensitivity of the first instability of the cylinder wake. J. Fluid Mech. 581, 167-197.

Hill, D.C., 1992. A theoretical approach for analyzing the re-stabilization of wakes. AIAA Paper, 92-0067.

Huerre, P., 2001. Open shear flow instabilities, in: Batchelor, G.K., Moffatt, H.K., Worster, M.G. (Eds.), Perspectives in Fluid Dynamics. Cambridge University Press, Cambridge (UK), pp. 159-229.

Huerre, P., Monkewitz, P.A., 1985. Absolute and convective instabilities in free shear layers. J. Fluid Mech. 159, $151-168$.

Huerre, P., Monkewitz, P.A., 1990. Local and global instabilities in spatially developing flows. Annu. Rev. Fluid Mech. 22, 473-537.

Huerre, P., Rossi, M., 1998. Hydrodynamic instabilities in open flows, in: Godrèche, C., Manneville, P. (Eds.), Hydrodynamic and Nonlinear Instabilities. Cambridge University Press, Cambridge (UK), pp. 81-294.

Hwang, Y., Choi, H., 2006. Control of absolute instability by basic-flow modification in a parallel wake at low reynolds number. J. Fluid Mech. 560, 465-475.

Hwang, Y., Choi, H., 2008. Sensitivity of global instability of spatially developing flow in weakly and fully nonlinear regimes. Phys. Fluid 20, 071703.

Jackson, C.P., 1987. A finite-element study of the onset of vortex shedding in flow past variously shaped bodies. J. Fluid Mech. 182, 23-45. 
Le Dizés, S., Huerre, P., Chomaz, J., Monkewitz, P., 1993. Nonlinear stability analysis of slowly varying medias: limitation of the weakly nonlnear approach, pp. 147-152.

Le Dizés, S., Huerre, P., Chomaz, J., Monkewitz, P., 1996. Linear global modes in spatially-developing media. Phil. Trans. R. Soc. Lond. A, 169-212.

Lesshafft, L., Huerre, P., Sagaut, P., Terracol, M., 2006. Nonlinear global modes in hot jets. J. Fluid Mech. 553, 393-409. Luchini, P., Giannetti, F., Pralits, J.O., 2008. Structural sensitivity of linear and nonlinear global modes. AIAA paper, 2008-4227.

Marquet, O., Sipp, D., Jacquin, L., 2008. Sensitivity analysis and passive control of cylinder flow. J. Fluid Mech. 615 221-252.

Mathis, C., Provansal, M., Boyer, L., 1984. The Bénard-von Kármán instability: an experimental study near the threshold. J. Phys. Paris Lett. 45, 483-491.

Meliga, P., Chomaz, J.M., Sipp, D., 2009. Global mode interaction and pattern selection in the wake of a disk: a weakly nonlinear expansion. J. Fluid Mech. 633, 159-189.

Monkewitz, P., Huerre, P., Chomaz, J., 1993. Global linear stability analysis of weakly non-parallel shear flows. J. Fluid Mech. 251, 1-20.

Pier, B., 2002. On the frequency selection of finite-amplitude vortex shedding in the cylinder wake. J. Fluid Mech. 458 407-417.

Pier, B., 2003. Open-loop control of absolutely unstable domains. Proc. R. Soc. Lond. A 459, 1105-1115.

Pier, B., Huerre, P., 1996. Fully nonlinear global modes in spatially developing media. Physica D 97, 206-222.

Pier, B., Huerre, P., 2001a. Nonlinear self-sustained structures and fronts in spatially developing wake flows. J. Fluid Mech. 435, 145-174.

Pier, B., Huerre, P., 2001b. Nonlinear synchronization in open flows. J. Fluids Struct. 15, 471-480.

Pier, B., Huerre, P., Chomaz, J.M., 2001. Bifurcation to fully nonlinear synchronized structures in slowly varying media. Physica D 148, 49-96.

Pier, B., Huerre, P., Chomaz, J.M., Couairon, A., 1998. Steep nonlinear global modes in spatially developing media. Phys. Fluids 10, 2433-2435.

Provansal, M., Mathis, C., Boyer, L., 1987. Bénard-von Kármán instability: Transient and forced regimes. J. Fluid Mech. $182,1-22$.

van Saarloos, W., 2003. Front propagation into unstable states. Phys. Rep. 386, 29-222.

Strykowski, P.J., Niccum, D.L., 1991. The stability of countercurrent mixing layers in circular jets. J. Fluid Mech. 227, 309-343.

Strykowski, P.J., Sreenivasan, K.R., 1990. On the formation and suppression of vortex 'shedding' at low Reynolds number. J. Fluid Mech. 218, 71-107.

Wang, Q., 2012. Forward and adjoint sensitivity computation for chaotic dynamical systems. J. Comp. Phys. $235,1-13$.

Zebib, A., 1987. Stability of viscous flow past a circular cylinder. J. Eng. Math. 21, 155-165. 